\title{
Bioestratigrafía y paleoambiente de la Formación Agrio (Cretácico Inferior), en la Provincia de Mendoza, Cuenca Neuquina, Argentina
}

Sara Ballent

Andrea Concheyro

Guillermina Sagasti
CONICET-Departamento Paleontología Invertebrados, Museo de Ciencias Naturales de La Plata, Paseo del Bosque s/n La Plata (1900), Argentina sballent@fcnym.unlp.edu.ar CONICET-Departamento de Ciencias Geológicas, Universidad de Buenos Aires, Pabellón II, Ciudad Universitaria (1428), Buenos Aires, Argentina IAA Instituto Antártico Argentino andrea@gl.fcen.uba.ar

Repsol-YPF Madrid, Paseo de la Castellana 280, Madrid, España guisagasti@yahoo.com

RESUMEN

Se caracteriza bioestratigráfica y paleoambientalmente a la Formación Agrio en la sección Cuesta del Chihuido, en

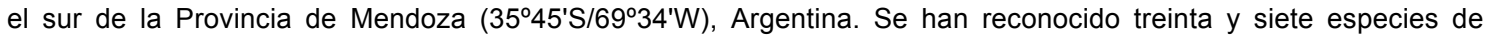
nanofósiles, treinta y cuatro de foraminíferos y seis de ostrácodos. Los nanofósiles permiten asignar la edad al Valanginiano superior-Hauteriviano superior e incluir la asociación en las Zonas CC4A y CC4B. En la base de la sección, la asociación del foraminífero bentónico Epistomina loncochensis Ballent y radiolarios sugieren condiciones altamente eutróficas en la columna de agua con abundante desarrollo de microbiota y fondos deficitarios en oxígeno. Hacia la parte media, la diversidad de foraminíferos lagénidos y de nanofósiles señala condiciones marinas normales de plataforma, con aguas claras y bien oxigenadas. Abundantes polimorfínidos, spirillínidos e involutínidos hacia el techo de la sección indican una marcada tendencia a la somerización. Las intercalaciones calcáreas-pelítico arenosas que representan depósitos tempestíticos distales contienen una microfauna alóctona de foraminíferos adheridos y caparazones adultos de ostrácodos cytheroideos.

Palabras claves: Cretácico Inferior, Formación Agrio, Bioestratigrafía, Paleoambiente, Microfósiles calcáreos, Argentina.

\section{ABSTRACT}

Biostratigraphy and palaeoenvironment of the Agrio Formation (Lower Cretaceous), Mendoza Province, Neuquén Basin, Argentina. A bioestratigraphical and palaeoenvironmental study of the Agrio Formation, at the Cuesta del Chihuido section, southern Mendoza Province ( $\left.35^{\circ} 45^{\prime} \mathrm{S} / 69^{\circ} 34^{\prime} \mathrm{W}\right)$, Argentina, is presented. Thirty seven species of nannofossils, thirty four of foraminifers and six of ostracods have been recognized. Nannofossils association corresponding to Zones CC4A y CC4B indicates an Upper Valanginian-Upper Hauterivian age. At the base of the section, the presence of the benthonic foraminifer Epistomina loncochensis Ballent and radiolaria suggests eutrophic conditions of surface waters and oxygen depressed sea-bottom conditions. In the middle part of the section, diversity of lagenid foraminifers and nannofossils indicates a shelf and nearshore environment with clear and well-oxygenated waters. 
Abundant polymorphinids, spirillinids and involitinids towards the top of the section corroborate a general regressive tendency. Mudstones and silty-sandstone intercalations containing allochthonous adherent foraminifers and adult carapaces of cytheroid ostracods represent distal storm deposits.

Key words: Lower Cretaceous, Agrio Formation, Biostratigraphy, Palaeoenvironment, Calcareous microfossils, Argentina.

\section{INTRODUCCIÓN Y ANTECEDENTES}

La Formación Agrio constituye una unidad litoestratigráfica de amplia distribución en la Cuenca Neuquina del centro oeste de Argentina, que comprende la porción terminal del Grupo Mendoza (Stipanicic et al., 1968), el cual comienza en el Kimmeridgiano y culmina en el Barremiano inferior. Clásicamente, la Formación Agrio ha sido dividida en tres miembros: Miembro Agrio inferior, Miembro Avilé y Miembro Agrio superior, pero, dado que las denominaciones de Agrio inferior y Agrio superior no cumplían con el Código Argentino de Nomenclatura Estratigráfica, Leanza y Hugo (2001) propusieron las nominaciones Miembro Pilmatué, Miembro Avilé y Miembro Agua de la Mula, respectivamente. Los miembros Pilmatué y Agua de la Mula afloran extensamente en toda la cuenca y están constituidos por pelitas, calizas y en menor proporción areniscas, depositadas durante episodios transgresivos y de nivel de mar alto. El Miembro Avilé es de naturaleza silicoclástica y constituye un evento de desecación instantánea de la cuenca (Leanza y Hugo, 2001, p. 31) o una regresión forzada (Rossi, 2001) y sólo se depositó en los sectores internos de la cubeta, durante un episodio de descenso relativo del nivel del mar.

La Formación Agrio es, en diferentes localidades, la que ha aportado las microfaunas más diversas y si bien las mismas han sido descritas e ilustradas en diversas publicaciones, no se cuenta con contribuciones que relacionen los aspectos bioestratigráficos con los microfósiles calcáreos y su vinculación con el contexto sedimentológico.

En la presente contribución se realiza el análisis bioestratigráfico de la Formación Agrio en la sección Cuesta del Chihuido, en el sudoeste de la Provincia de Mendoza, Argentina (Fig. texto 1) utilizando la biozonación basada en nanofósiles propuesta por Bown y Concheyro (2004) y refiriéndose además a otros microfósiles calcáreos bentónicos asociados, tales como los foraminíferos y ostrácodos. El objetivo de este estudio consiste en caracterizar a la Formación Agrio en este sector de la Cuenca Neuquina de Argentina a partir de su contenido en microfósiles calcáreos, de las inferencias paleoambientales a partir de éstos y de las características del sedimento portador. El estudio sedimentológico de la unidad es parte de la tesis doctoral de una de las autoras (Sagasti, 2002).

Entre los antecedentes se menciona a Simeoni y Musacchio (1996) y Simeoni $(2000,2001)$ quienes se han referido a los microfósiles de esta localidad, describiendo solamente algunos foraminíferos, listando los nanofósiles calcáreos y reconociendo biohorizontes basados en el contenido de foraminíferos bentónicos.

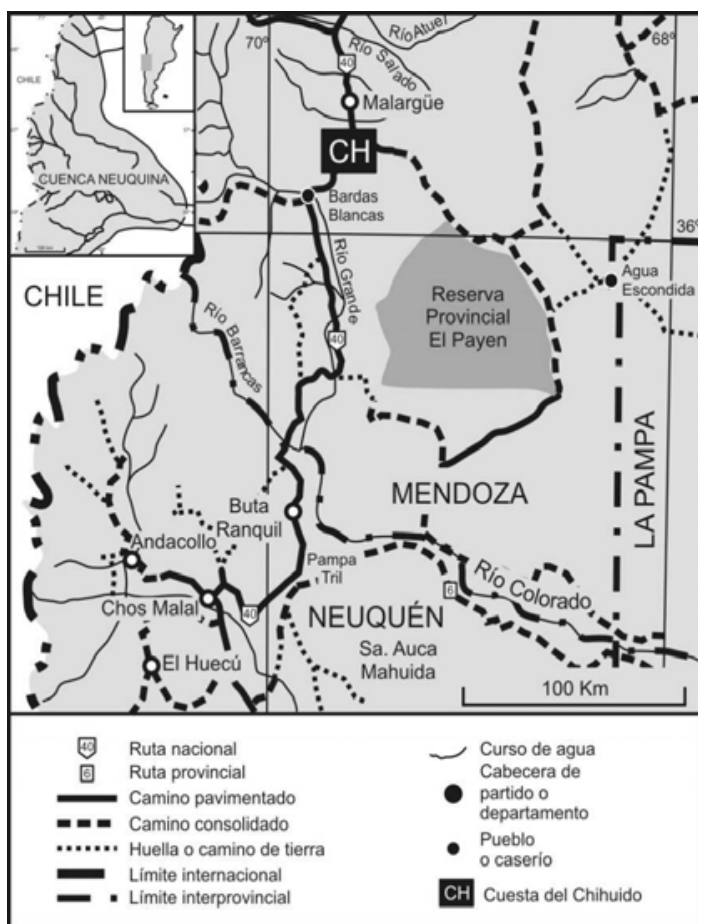

FIG. texto1. Cuenca Neuquina, centro oeste de Argentina; ubicación de la localidad Cuesta del Chihuido. 
Foraminíferos y ostrácodos. En la sistemática de foraminíferos se utilizó la clasificación propuesta por Loeblich y Tappan (1988) y para ostrácodos la propuesta en Moore y Pitrat (1961). Medidas y abreviaturas: en foraminíferos, $\mathrm{L}=$ longitud , $a=$ ancho, $\mathrm{h}=$ altura, $\mathrm{Dm}=$ diámetro menor, $\mathrm{DM}=$ diámetro mayor, $\mathrm{E}$ (altura en plano axial)= espesor; en ostrácodos, $L=$ largo, $A=$ alto, $a=$ ancho. Las dimensiones se indican en milímetros $(\mathrm{mm})$.

Repositorio. Los nanofósiles ilustrados han sido depositados en las colecciones del Departamento de Ciencias Geológicas de la Facultad de Ciencias Exactas y Naturales de la Universidad de Buenos Aires bajo la sigla BAFC-NP (Nanoplancton calcáreo), numeraciones 2250 a 2272 . El material de foraminíferos y ostrácodos se encuentra depositado en el Departamento Paleozoología Invertebrados (Sección Micropaleontología) del Museo de Ciencias Naturales de La Plata, Argentina, bajo la sigla MLP-Mi 1424-1534.

\section{ÁREA DE ESTUDIO Y DESCRIPCIÓN DE LA SECCIÓN ESTUDIADA}

La Cuesta del Chihuido se encuentra en el sur de la Provincia de Mendoza, a los $35^{\circ} 45^{\prime} \mathrm{S}$ y $69^{\circ}$ 34 'S, entre las localidades de Malargüe y Bardas Blancas (Fig. texto 1). El perfil fue medido sobre los afloramientos contiguos a la ruta Nacional No. 40. En esta localidad están presentes los Miembros Pilmatué y Agua de la Mula de la Formación Agrio, en tanto que el Miembro Avilé no está representado.

La secuencia tiene $205 \mathrm{~m}$ de espesor y se compone principalmente de pelitas negras, margas y calizas micríticas. El límite inferior es concordante y neto, y se ubica entre las calizas con ostras de la Formación Chachao y las calizas micríticas con foraminíferos bentónicos (Epistomina loncochensis Ballent), características de la base de la Formación Agrio. La Formación Huitrín yace en concordancia sobre la Formación Agrio y el pasaje entre estas entidades se ha ubicado donde aparecen los primeros bancos de caliza con fauna de ambiente marino somero (foraminíferos polimorfínidos y patellínidos), correspondientes a la Formación Huitrín (Fig. texto 2).

\section{ANÁLISIS SEDIMENTOLÓGICO Y CONTENIDO FOSILÍFERO}

Sobre la base de los atributos macroscópicos, tales como litología, diseño de apilamiento, estructuras sedimentarias primarias inorgánicas y biogénicas y el contenido fósil, la sucesión de la Formación Agrio ha sido dividida en seis intervalos (Fig. texto 2):

Intervalo 1: se trata de un paquete de $47,60 \mathrm{~m}$ de potencia caracterizado por el predominio de pelitas negras y margas con intercalaciones aisladas de calizas micríticas con abundantes foraminíferos bentónicos y trazas fósiles de Thalassinoides. En la porción superior de este intervalo se reconocen algunos niveles de calizas nodulares.

Intervalo 2: posee $18,60 \mathrm{~m}$ de espesor y está constituido por intercalaciones rítmicas de pelitas negras, margas y calizas. De la base al techo, se observa que las pelitas y margas muestran una tendencia estratodecreciente, comenzando con capas de 1,40 m de espesor y llegando a conformar niveles de no más de $0,20 \mathrm{~m}$. El espesor de las calizas es uniforme, con valores muy frecuentes en torno a los $0,20 \mathrm{~m}$, y extremos de 0,15 y 0,60 metros. Los bancos de caliza del tramo inferior están desprovistos de fauna, en tanto que aquellos correspondientes a la sección superior contienen bivalvos y gastrópodos; además, presentan rasgos de bioturbación en su base.

Intervalo 3: tiene 24,60 m de espesor representados por intercalaciones de margas y calizas, con una tendencia general a la disminución en el espesor de las margas en sentido ascendente (desde 5,90 hasta $0,25 \mathrm{~m}$ ). Las calizas no muestran variaciones significativas de espesor, en general poseen entre 0,20 y $0,60 \mathrm{~m}$ de potencia. En la mayor parte de los bancos de caliza se hallaron restos de bivalvos y amonites y se reconoció bioturbación.

Los intervalos 1, 2 y 3 corresponden al Miembro Pilmatué de la Formación Agrio.

Intervalo 4: abarca un tramo potente de la sucesión (56,60 m) caracterizado por el desarrollo de ritmos marga-caliza con una ligera tendencia estratodecreciente. A excepción del primer nivel margoso que tiene 2,60 $\mathrm{m}$ de espesor, el resto no supera los $1,50 \mathrm{~m}$ de potencia (con un promedio de 0,30 


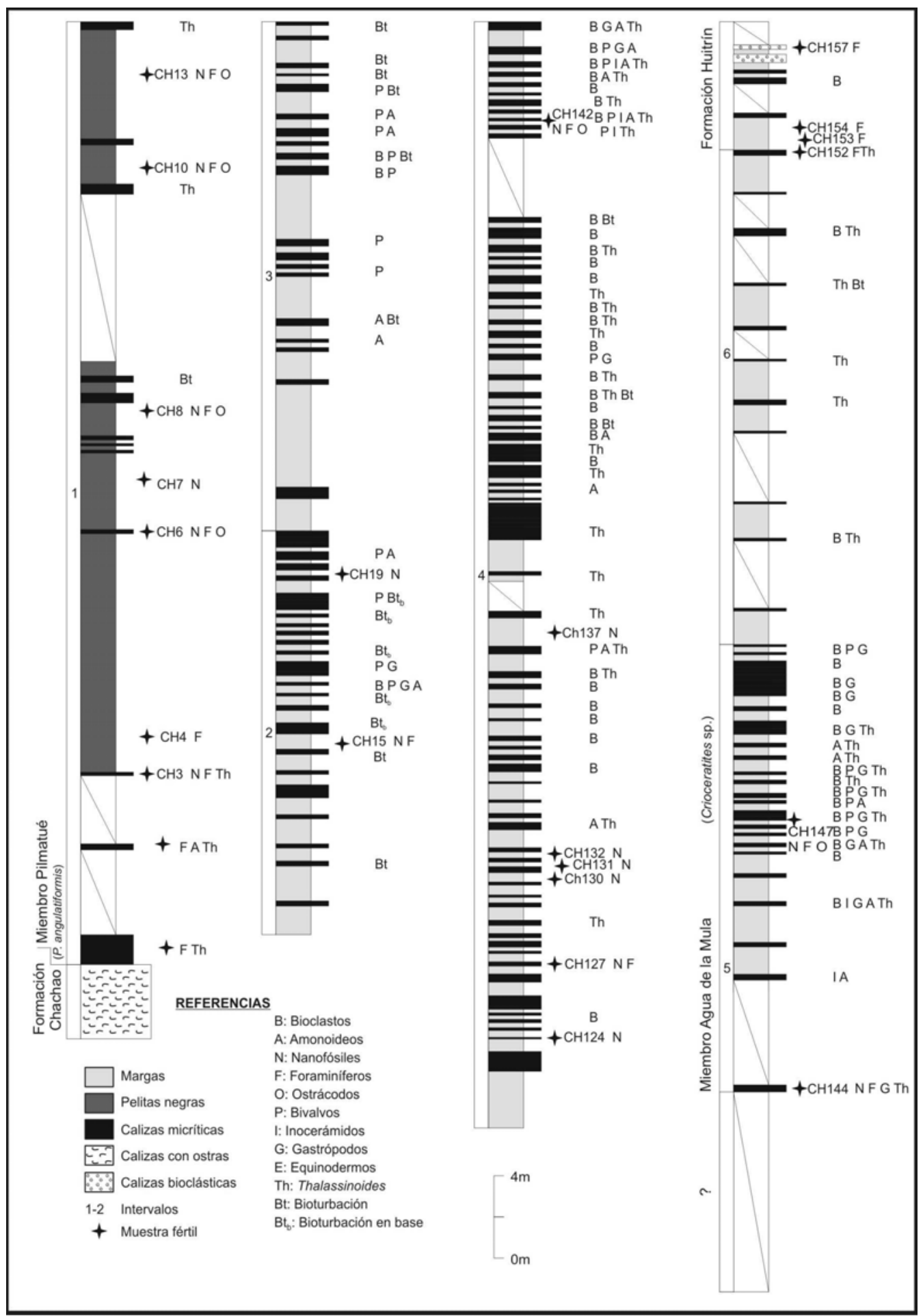

FIG. texto 2. Perfil de la sección Cuesta del Chihuido, Cuenca Neuquina, Argentina. Contexto sedimentológico y contenido fosilífero. 
metros). Los bancos de caliza presentan un espesor medio de $0,20 \mathrm{~m}$ y contienen fragmentos esqueletales no diferenciables y restos de bivalvos Inoceramus sp., gastrópodos y amonites (Holcoptychites sp.) así como también trazas fósiles (Thalassinoides).

Intervalo 5: se trata de un conjunto rítmico constituido por intercalaciones de margas y calizas que tiene $31,40 \mathrm{~m}$ de potencia. Se aprecia un claro arreglo estratodecreciente definido por la variación en el espesor de las margas, desde potentes bancos de $5 \mathrm{~m}$ hasta delgados niveles de 0,20 m. Las calizas poseen espesores muy uniformes de entre 0,15 y $0,35 \mathrm{~m}$ y se caracterizan por su elevado contenido fósil, representado por fragmentos de organismos no diferenciados, bivalvos (Inoceramus $\mathrm{sp}$.) y amonites (Crioceratites sp.); además son frecuentes las trazas de Thalassinoides.

Intervalo 6: comprende los últimos $28 \mathrm{~m}$ de la sucesión. Se compone de ritmos marga-caliza caracterizados por presentar niveles margosos de espesor relativamente uniforme, con valores comprendidos entre 1,20 y 1,80 m. Las capas de caliza tienen entre 0,10 y $0,30 \mathrm{~m}$ de potencia y poseen escasos restos de organismos no diferenciados y trazas de Thalassinoides.

Los intervalos 4, 5 y 6 corresponden al Miembro Agua de la Mula de la Formación Agrio.

Cabe destacar que a lo largo de la sucesión de la Formación Agrio se han reconocido intercalaciones esporádicas de niveles calcáreos pelíticoarenosos de entre 0,05 y 0,25 m de espesor. Dichos niveles presentan contactos netos, en partes con techo ondulado y se encuentran moderada a profusamente bioturbados. En los niveles menos afectados por bioturbación es frecuente observar gradación normal o laminación horizontal. En estos horizontes se ha podido observar que la microfauna está caracterizada por el predominio de ejemplares transportados.

\section{COMPOSICIÓN DE LA MICROFAUNA}

La microfauna recuperada está representada por nanofósiles, foraminíferos y ostrácodos y los radiolarios spumelláridos los que tienen una presencia constante y abundante a través de toda la sección. En algunos niveles son francamente abundantes piezas desarticuladas de equinodermos, tales como osículos vertebrales de ofiuroideos, escleritos de holoturoideos y espinas de equinoideos. La distribución de los distintos restos recuperados se indica en la figura texto 2 . Nanofósiles calcáreos: se han recuperado nanofósiles calcáreos en diecisiete muestras de las veintitrés procesadas; se siguió la técnica de rutina de extendidos microscópicos o 'smear slides' (Edwards, 1963; Bown y Young, 1998). Las observaciones se realizaron con un microscopio Leitz de polarización, efectuándose un estudio cualitativosemicuantitativo; se revisaron tres transectas longitudinales, siguiendo el eje mayor del preparado y se identificaron los nanofósiles marcadores y el resto de la asociación. La distribución de los nanofósiles calcáreos y los criterios de abundancia se indican en la figura texto 3.

Se han contabilizado 37 especies de nanofósiles, en su mayoría cosmopolitas. La preservación de los ejemplares varía de moderada a buena. En ciertos niveles, Nannoconus steinmannii Kampter, Nannoconus quadricanalis Bown y Concheyro y Watznaueria barnesae (Black y Barnes) son las especies predominantes.

Foraminíferos: se ha recuperado un total de treinta y cuatro especies, las que están irregularmente distribuidas a lo largo de la sección (Fig. texto 4). Los niveles inferiores (muestras $\mathrm{CH} 3,4)$ son portadores exclusivamente de Epistomina loncochensis Ballent, especie característica del intervalo basal con que se inicia la depositación de la Formación Agrio en el sector septentrional de la Cuenca Neuquina. Entre las muestras $\mathrm{CH} 6$ y 142 se han recuperado treinta y tres especies, de las cuales cinco corresponden a Textulariina (pared aglutinada), y el resto a tres subórdenes (Lagenina, Spirillinina e Involutinina) de pared calcárea.

De los taxones aglutinados, Sculptobaculites sp. es el más abundante; éste, junto a los adherentes Tolypammina? sp. y Ammovertella? sp., y a un lagénido de igual hábito, Bullopora laevis (Sollas), están concentrados en la muestra $\mathrm{CH}$. Otro taxón de hábito adherido Webbinella? sp., está presente en la muestra $\mathrm{CH} 147$. 


\begin{tabular}{|c|c|c|c|c|c|c|c|c|c|c|c|c|c|c|c|c|c|c|c|c|c|c|c|c|}
\hline \multicolumn{11}{|c|}{ Fm. Agrio-Miembro Pilmatué } & \multicolumn{11}{|c|}{ Fm. Agrio-Miembro Agua de la Mula } & \multicolumn{2}{|c|}{ F. Huitrín } & \multirow{2}{*}{$\begin{array}{c}\text { FORMACIONES } \\
\text { MUESTRAS }\end{array}$} \\
\hline$\frac{\rho}{\mathcal{T}}$ & $\frac{\Omega}{\frac{\rho}{x}}$ & 莖 & 옹 & $\stackrel{?}{\text { 工 }}$ & 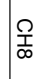 & 畄 & $\frac{\text { ? }}{\text { I }}$ & $\frac{\stackrel{\rho}{I}}{\vec{\omega}}$ & 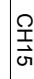 & $\frac{\stackrel{\rho}{I}}{\vec{\omega}}$ & 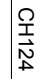 & & 畡 & 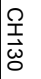 & 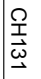 & 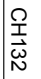 & 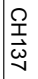 & 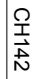 & 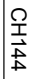 & 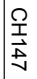 & 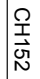 & 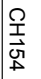 & $\frac{\stackrel{\Omega}{I}}{\mathrm{v}}$ & \\
\hline 3 & & & 3 & 3 & 3 & & $\infty$ & - & - & 3 & 3 & & 3 & 3 & 3 & 3 & 3 & & 0 & 0 & & & & PRESERVACIÓN \\
\hline ס & & & 0 & $\pi$ & $\pi$ & & $>$ & $\pi$ & $\pi$ & $>$ & $>$ & & $>$ & $>$ & $\pi$ & $>$ & $>$ & & 历 & ס & & & & ABUNDANCIA \\
\hline \multirow{38}{*}{\multicolumn{2}{|c|}{0}} & & ס & D & $\pi$ & & $>$ & $\pi$ & $\pi$ & $>$ & $\pi$ & & $>$ & $>$ & $\pi$ & $>$ & $>$ & ס & ס & ס & & & & Watznaueria barnesae \\
\hline & & & & ס & & & & & ס & & $\pi$ & & $\pi$ & & & $\pi$ & $\pi$ & & & & & & & Zeughrabdotus embergeri \\
\hline & & & & ס & & & ס & & & ס & ס & & סע & & & & & ס & & & & & & Rhagodiscus asper \\
\hline & & & & ס & & & $\varnothing$ & & & & & & & & & & & & & & & & & Zeughrabdotus erectus \\
\hline & & & & 0 & & & $\pi$ & $\pi$ & ס & ס & $\pi$ & & & ס & 0 & 力 & & 力 & & & & & & Cyclagelosphaera margerelii \\
\hline & & & & ס & & & & & & & & & & & & & & & & & & & & Manivitella pemmatoidea \\
\hline & & & & $\pi$ & & & ס & & & & & & & & & & & & & & & & & Tranolithus phacelosus \\
\hline & & & & $\pi$ & & & $\pi$ & ס & & 力 & ס & & & $\pi$ & & ס & 力 & 力 & & & & & & Micrantholithus hoschulzii \\
\hline & & & & ס & & & & & & & & & & & $\pi$ & & & & & & & & & Nannoconus sp. \\
\hline & & & & ס & ס & & $\varnothing$ & & & & & & & & & & & & & & & & & Retecapsa crenulata \\
\hline & & & & 0 & & & $\pi$ & $\varnothing$ & ס & 力 & $\pi$ & & $\pi$ & 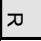 & & & & & & & & & & Eiffellithus striatus \\
\hline & & & & ס & & & & & & & & & & & & & & & & & & & & Stradnerlithus silvaradius \\
\hline & & & & & & & ס & ס & ס & ס & & & & & & & & & & & & & & Diazomatholithus lehmannii \\
\hline & & & & & & & $\pi$ & ס & D & $\pi$ & $>$ & & $\varnothing$ & $>$ & $\varnothing$ & & $\varnothing$ & $\varnothing$ & & $\varnothing$ & & & & Watzanueria fossacincta \\
\hline & & & & & & & $\varnothing$ & & & & & & & & & & & & & & & & & Watznaueria barnesae (cocósfera) \\
\hline & & & & & & & & ס & & & & & & & & & & & & & & & & Watznaueria biporta \\
\hline & & & & & & & $\varnothing$ & & ס & ס & ס & & ס & & & & & & & & & & & Ellipsagelosphaera britannica \\
\hline & & & & & & & $\varnothing$ & & & & & & & $\pi$ & & & & & & & & & & Micrantholithus obtusus \\
\hline & & & & & & & & ס & & & & & ס & ס & & $\pi$ & & & & & & & & Ellipsagelosphaera ovata \\
\hline & & & & & & & & ס & & & ס & & $\pi$ & $\pi$ & & ס & ס & & & & & & & Retecapsa surirella \\
\hline & & & & & & & & ס & ס & ס & & & & & & & & & & & & & & Eiffellithus windii \\
\hline & & & & & & & & & & & & & & & & & & & & & & & & Watznaueria biporta \\
\hline & & & & & & & & & & ס & ס & & ס & 0 & & & ס & & & ס & & & & Nannoconus circularis \\
\hline & & & & & & & & & & $\pi$ & & & & & ס & & $\pi$ & 力 & & & & & & Nannoconus steinmannii \\
\hline & & & & & & & & & & ס & & & 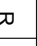 & & ס & & o & & & & & & & Nannoconus quadricanalis \\
\hline & & & & & & & & & & ס & ס & & ס & & & $\varnothing$ & & & & & & & & Micrantholithus obtusus \\
\hline & & & & & & & & & & & & & 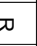 & & & & & & & & & & & Haqius circumradiatus \\
\hline & & & & & & & & & & & $\pi$ & & ס & & & & & & & & & & & Cretarhabdus conicus \\
\hline & & & & & & & & & & & & & ס & & & & & & & & & & & Cruciellipsis cuvillieri \\
\hline & & & & & & & & & & & & & ס & & & & & & & & & & & Helenea chiasta \\
\hline & & & & & & & & & & & & & ס & & & & & & & & & & & Zeughrabdotus sp. \\
\hline & & & & & & & & & & & ס & & & & & & & & & & & & & Lithraphidites bollii \\
\hline & & & & & & & & & & ס & ס & & & & & & & & & & & & & Nannoconus bucheri \\
\hline & & & & & & & & & & ס & & & & & & & & & & & & & & Stradnerlithus comptus \\
\hline & & & & & & & & & & ס & & & & & & & & & & & & & & Staurolithites sp. \\
\hline & & & & & & & & & & ס & & & & & & & & & & & & & & Nannoconus kamptneri \\
\hline & & & & & & & & & & & ס & & & & & & & & & & & & & Calculites suturus \\
\hline & & & & & & & & & & ס & & & & & & & & & & & & & & Lithraphidites carniolensis \\
\hline
\end{tabular}

FIG. texto 3. Carta de distribución de las especies de nanofósiles calcáreos halladas en la sección Cuesta del Chihuido, Cuenca Neuquina, Argentina. Preservación. P: pobre; M: moderada, B: buena. Abundancia; P: presente, con un espécimen cada 10 ó más campos de observación; $\mathbf{R}$ : rara, con un espécimen cada 1 a 10 campos; $\mathbf{F}$ : frecuente, con un espécimen cada campo de observación; C: común; 1-5 especímenes por cada campo de observación; A: abundante, más de 5 especímenes por cada campo de observación. 


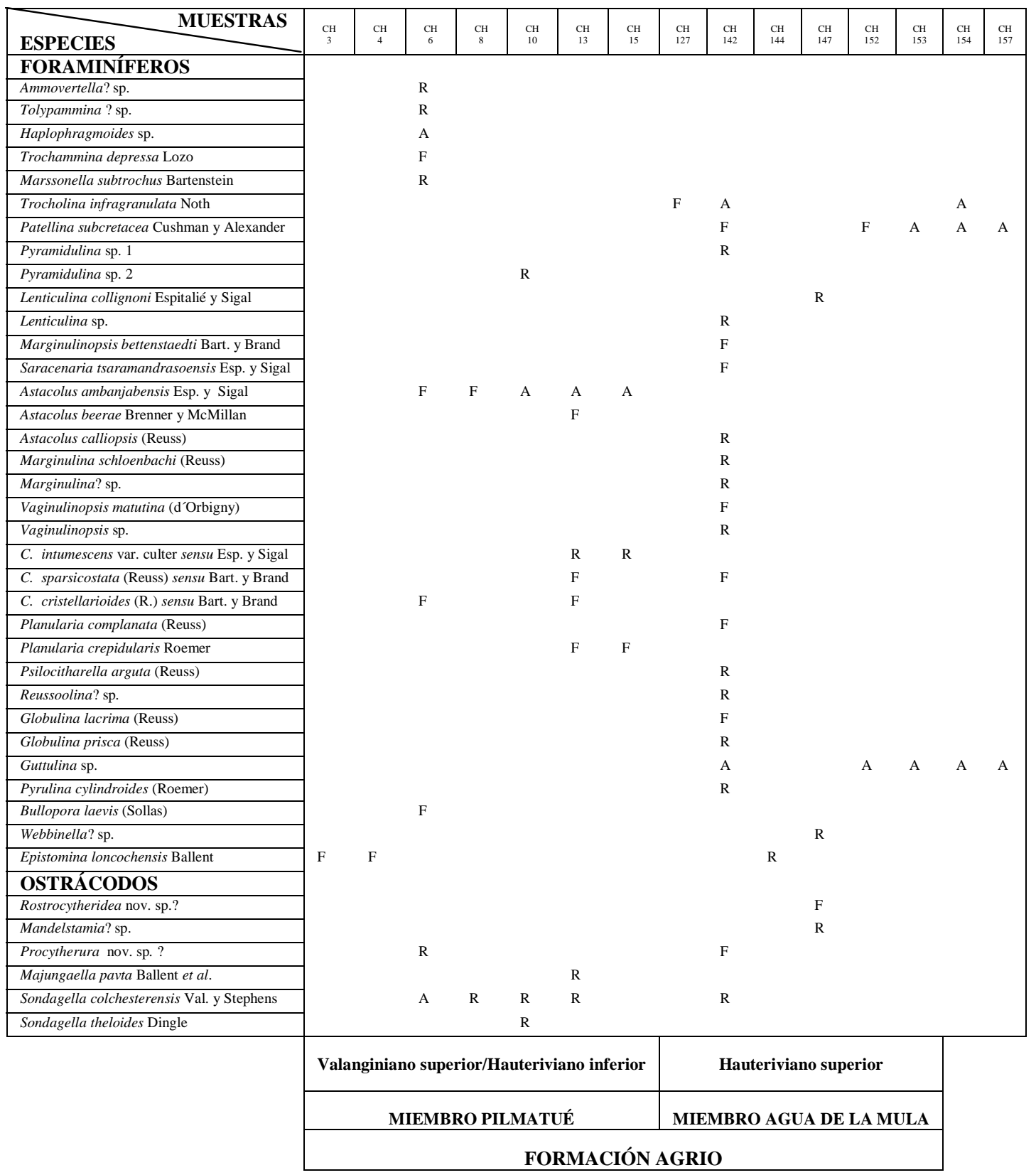

FIG. texto 4. Distribución y abundancia relativa (aproximadamente en 30 gramos de muestra lavada) de foraminíferos y ostrácodos en la sección Cuenta del Chihuido, Cuenca Neuquina, Argentina. A= abundante (más de 50 especímenes); $F=$ frecuente (entre 20 y 50 especímenes); $\mathrm{R}=$ raro (menos de 5 especímenes).

En el techo de la sección, muestras $\mathrm{CH} 152$ a 157, en transición a la Formación Huitrín suprayacente, dominan pequeñas conchillas de forma cónica: Trocholina infragranulata Noth (Involutinina) y Patellina subcretacea Cushman y Alexander
(Spirillinina, Patellinidae), además de polimorfínidos tales como Guttulina sp.

Ostrácodos: los ostrácodos están mayoritariamente presentes en las muestras $\mathrm{CH} 6,7,10,13 \mathrm{y}$ 147 (Fig. texto 4), con seis especies de Cytheroidea 
comprendidas en los géneros Rostrocytheridea Dingle, Mandelstamia? Ljubimova, Procytherura Whatley, Majungaella Grekoff y Sondagella Dingle. Sondagella colchesterensis Valicenti y Stephens, con más de 500 ejemplares en la muestra $\mathrm{CH} 6$, es la más abundante. Excepcionalmente, se hallaron una valva derecha de Cytherella Jones (en la muestra $\mathrm{CH} 6$ ) y un caparazón juvenil de Cytherella amosi Musacchio (en la muestra $\mathrm{CH} 147$ ). En cortes delgados de las muestras $\mathrm{CH} 152$ y 153 , ya dentro de la Formación Huitrín, se reconocieron frecuentes valvas de ostrácodos lisos de filiación marina (Paracypris?), aunque no se recuperaron durante la búsqueda bajo lupa binocular.

\section{BIOESTRATIGRAFÍA-NANOFÓSILES CALCÁREOS}

El perfil estudiado es portador de una asociación relativamente bien preservada de nanofósiles calcáreos asignables al Valanginiano-Hauteriviano. La misma posee una moderada diversidad y en algunas muestras resulta abundante en cantidad de ejemplares recuperados.

Las primeras muestras correspondientes a la base del perfil resultan estériles, posiblemente debido a problemas de disolución, asociados a fondos poco oxigenados y condiciones altamente reductoras; esta situación se observó en otras localidades de la Cuenca Neuquina y en el mismo intervalo de la Formación Agrio. Las tres últimas muestras del perfil resultan estériles dado que éstas fueron recolectadas en estratos de la Formación Huitrín, estéril en nanofósiles, al menos hasta el momento (Figs. texto 2, 3).

Desde el punto de vista bioestratigráfico se han reconocido los siguientes bioeventos: la FO (primera aparición) y LO (última aparición) de Eiffellithus striatus (Black), la FO de Nannoconus circularis Deres y Achéritéguy y nanocónidos de canal ancho, la LO de Eiffellithus windii Applegate y Bergen, la presencia conjunta de Nannoconus bucheri Brönnimann y Lithraphidites bollii Thierstein y la LO de Cruciellipsis cuvillieri (Manivit) (véase Figs. texto 3, 5).

La FO de Eiffellithus striatus se registra en la muestra $\mathrm{CH} 7$, y es posible situarla en la Zona de amonites (ZA) de Pseudofavrella angulatiformis; cabe agregar que este amonite ha sido también hallado en la sección basal del perfil. La LO de Eiffellithus windii se registra en la muestra $\mathrm{CH} 19$ junto con la FO de Nannoconus circularis y los nanocónidos de canal ancho, y sugieren la posible transición entre la SA (Subzona de amonites) de Pseudofavrella angulatiformis a la SA de Holcoptychites neuquensis (Bown y Concheyro, 2004).
En particular en la muestra $\mathrm{CH} 20$, se identificaron ejemplares del ammonite Holcoptychites, por lo tanto el intervalo $\mathrm{CH}$-19 quedaría comprendido entre las SA de Chacantuceras ornatum y de Holcoptychites neuquensis (Figs. texto 3, 5).

A partir de la muestra $\mathrm{CH} 19$ y hasta la $\mathrm{CH} 124$ no se han recolectado muestras para nanofósiles calcáreos, pero es posible que dicho intervalo constituya parte de una sección condensada que involucre también condensadamente las SA Olcostephanus laticostus y la BA (Biozona) Weavericeras vacaense. En otra región de la Provincia de Mendoza, se han reconocido todas las SA para el Miembro Pilmatué de la Formación Agrio (B. Aguirre-Urreta, comunicación oral, 2004).

Tomando en consideración los nanofósiles calcáreos hallados en el intervalo $\mathrm{CH} 3-\mathrm{CH} 19$ de la sección Cuesta del Chihuido, es posible asignar al Miembro Pilmatué de la Formación Agrio una edad valanginiana superior a hauteriviana inferior (Fig. texto 5).

A partir de la muestra $\mathrm{CH} 124$ se identifica claramente el Miembro Agua de la Mula sobre la base de sus nanofósiles. La presencia conjunta en esta muestra de Nannoconus bucheri y Lithraphidites bollii permite situar la muestra en la BA de Spitidiscus riccardii. La LO de Cruciellipsis cuvillieri en $\mathrm{CH} 127$ precisa el pasaje de la BA de S. riccardii a la BA de Crioceratites schlagintweiti (Figs. texto $3,5)$.

La LO Eiffellithus striatus se registra en la muestra $\mathrm{CH} 130$, que permite ubicar a la misma en la BA de Crioceratites schlagintweiti.

Hacia el techo de la secuencia se recuperaron algunos ejemplares fragmentarios del amonite Crioceratites diamantensis; en este intervalo los nanofósiles resultan escasos. Se trata de una asociación monótona que no registra especies 


\begin{tabular}{|c|c|c|c|}
\hline \multirow{2}{*}{$\stackrel{0}{\infty}$} & \multicolumn{3}{|c|}{ CUENCA NEUQUINA } \\
\hline & $\begin{array}{c}\text { BIOZONAS AMONITES(B)/ } \\
\text { SUBZONAS (S) }\end{array}$ & EVENTOS NANOFOSILES & BIOZONAS \\
\hline$\infty$ & Paraspiticeras groeberi $\mathrm{B}$ & \multirow{2}{*}{$\begin{array}{l}\text { Nannoconus ligius } \\
\mathbf{X} \text { Lithraphidites bollii } \\
\text { Nannoconus ligius }\end{array}$} & \multirow{2}{*}{ CC5A } \\
\hline \multirow{3}{*}{ 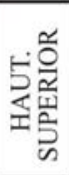 } & Crioceratites diamantensis $\mathrm{B}$ & & \\
\hline & Crioceratites schlagintweiti B & Eifellithus striatus & \multirow{2}{*}{$\mathrm{CC} 4 \mathrm{~B}$} \\
\hline & Spitididiscus riccardii B & Cruciellipsis cuvillieri & \\
\hline \multirow{5}{*}{ 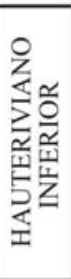 } & Weavericeras vacaense B & $\begin{array}{l}\text { Lithraphidites bollii } \\
\nabla \text { Clepsilithus maculosus }\end{array}$ & \multirow{8}{*}{$\mathrm{CC} 4 \mathrm{~A}$} \\
\hline & $\begin{array}{l}\text { Hoplitocrioceras gentilii } \mathrm{S} \\
\text { Hoplitocrioceras giovinei } \mathrm{S}\end{array}$ & & \\
\hline & Olcostephanus (O.) laticostus S & & \\
\hline & Holcoptychites agrioensis $\mathrm{S}$ & A Clepsilithus maculosus & \\
\hline & Holcoptychites neuquensis S & Fifellithus windii & \\
\hline \multirow{5}{*}{ 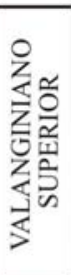 } & Neocomites $\mathrm{sp} . \mathrm{S}$ & & \\
\hline & Chacantuceras ornatum $\mathrm{S}$ & conus canal ancho & \\
\hline & Pseudofavrella angulatiformis $\mathrm{S}$ & onus canar ancio & \\
\hline & O. (Viluceras) permolestus $\mathrm{S}$ & Eifellithus striatus & \multirow{4}{*}{ CC3B } \\
\hline & Karakaschiceras attenuatus S & & \\
\hline \multirow{3}{*}{ 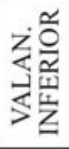 } & Olcostephanus (O.) atherstoni $\mathrm{S}$ & \multirow{3}{*}{$\begin{array}{c}\text { Eifellithus primus } \\
\text { Eifellithus windii } \\
\text { Eifellithus primus }\end{array}$} & \\
\hline & Lissonia riveroi $\mathrm{B}$ & & \\
\hline & Neocomites wichmanni B & & \multirow{3}{*}{$\mathrm{CC} 2$} \\
\hline \multirow{2}{*}{ 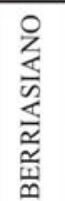 } & Spiticeras damesi $\mathrm{B}$ & \ Haqius circumradiatus & \\
\hline & Argentiniceras noduliferum $\mathrm{B}$ & $-? \boldsymbol{A}$ Cruciellipsis cuvillieri & \\
\hline
\end{tabular}

FIG. texto 5. Biozonas de amonites y bioeventos de nanofósiles calcáreos definidos para el Cretácico Inferior de la Cuenca Neuquina (tomado de Bown y Concheyro, 2004; zonación de amonites de Aguirre-Urreta y Rawson, 1997 y Aguirre-Urreta, 2001). En gris sombreado, biozonas reconocidas en la Formación Agrio, perfil Cuesta del Chihuido, Provincia de Mendoza, Argentina.

biomarcadoras y que por su posición estratigráfica es asignada a la BA de Crioceratites diamantensis del Hauteriviano superior.

Todos estos bioeventos definidos precedente- mente (Bown y Concheyro, 2004) permiten asignar el Miembro Agua de la Mula de la Formación Agrio en esta localidad al Hauteriviano superior.

\section{CONSIDERACIONES PALEOAMBIENTALES Y DE EDAD}

El paleoambiente de sedimentación de la Formación Agrio en la Cuenca Neuquina ha sido interpretado en sus rasgos generales por diversos autores. Mitchum y Uliana (1985), Legarreta y
Gulisano (1989) y Legarreta y Uliana (1991) establecieron que los sistemas deposicionales se desarrollaron en una amplia rampa sin clinoformas, caracterizada por la agradación de depósitos 
terrígenos y calcáreos. Más recientemente, Spalletti et al. (2001) analizaron en detalle los depósitos del Miembro Agua de la Mula, aflorantes en el area septentrional de la provincia del Neuquén, unos $200 \mathrm{~km}$ al sur de la Cuesta del Chihuido. Estos autores propusieron un modelo de rampa marina abierta, caracterizada por la acumulación de sedimentitas de grano fino en la zona de rampa externa a cuenca, con participación subordinada de limos, areniscas y bioconstrucciones carbonáticas, depositadas en la zona de rampa media a interna.

La característica sedimentológica de los Miembros Pilmatué y Agua de la Mula aflorantes en la Cuesta del Chihuido, tal como la presencia de depósitos suspensivos de fangos terrígenos y carbonáticos, sugieren un ambiente de acumulación marino relativamente profundo, localizado por debajo del nivel de base de olas de tormenta, en las zonas de rampa externa a cuenca (Burchette y Wright, 1992; Tucker, 1991; Tucker et al., 1993). Las intercalaciones calcáreas pelítico-arenosas, portadoras de estructuras tractivas y microfauna alóctona, se atribuyen a esporádicos episodios de mayor energía, los cuales se han intepretado como eventos de tormenta. De este modo, dichas intercalaciones representan depósitos tempestíticos distales.

Si bien los $10 \mathrm{~m}$ basales del perfil están parcialmente cubiertos, son diferenciables tres niveles de calizas micríticas de $1,40 \mathrm{~m}, 0,30 \mathrm{~m}$ y $0,20 \mathrm{~m}$ de espesor, respectivamente (Fig. texto 2 ). Este paquete basal contiene en forma dominante el foraminífero Epistomina loncochensis Ballent y abundantes radiolarios espumeláridos. Este conspicuo banco en la base del Miembro Pilmatué se visualiza en el sector norte de la Cuenca Neuquina con una continuidad lateral cercana a los $300 \mathrm{~km}$. Un análisis detallado y una discución sobre sus atributos sedimentológicos y paleontológicos figuran en Sagasti y Ballent (2002). El mismo se asocia con el evento transgresivo con que se inicia la deposición de la Formación Agrio en este sector de la cuenca, el que habría conducido a condiciones altamente eutróficas en la columna de agua, las cuales resultaron favorables para la proliferación y consiguiente abundancia de microbiota planctónica de radiolarios e inducido a condiciones de fondo deficitarias en oxígeno. Las características de la conchilla, plano convexa ó biconvexa, ventajosa para un modo de vida epifaunal o semiepifaunal (Koutsoukos et al., 1990, entre otros) y el hábito detritívoro activo en su alimentación parecen haber favorecido la expansión de E. loncochensis en niveles con tales condiciones.

Hacia arriba en el perfil (muestras $\mathrm{CH} 7$ a 142, abarcando el tercio medio y superior del Miembro Pilmatué y tercio inferior y medio del Miembro Agua de la Mula) se establecen condiciones marinas normales con fondos mejor oxigenados, demostrado por la presencia de una microfauna variada de foraminíferos Lagenina los cuales sugieren ambientes de plataforma marina con salinidad normal particularmente en áreas de sedimento de grano fino. La presencia de amonites, aunque escasos, y abundantes radiolarios, nanoplancton calcáreo y restos de equinodermos en este tramo de la sección, están indicando comunicación con el mar abierto. Consistentemente, la predominancia en algunos niveles de los nanofósiles Nannoconus steimannii Kamptner, Nannoconus quadricanalis Bown y Concheyro y Watznaueria barnesae (Black) indican condiciones ambientales marinas de plataforma, vinculadas con fondos bien oxigenados.

Entre los foraminíferos lagénidos, las formas más frecuentes son aquellas con conchillas biconvexas, con enroscamiento planospiral involuto y desenroscamiento hacia las cámaras finales, con periferia subaguda a carenada, tales como Astacolus ambanjabensis Espitalié y Sigal, Planularia crepidularis Roemer, Pravoslavlevia tsaramandrosoensis (Espitalié y Sigal) y especies de Lenticulina. En la cuenca cretácica de Sergipe, Brasil, Koutsoukos y Hart (1990) reúnen estas formas en el Submorfogrupo CH-A.6, adaptadas a un hábito epifaunal a semi-infaunal, cuyas conchillas lisas o poco ornamentadas y comprimidas sugieren haber sido rápidos cavadores euritópicos adaptados a ambientes de plataforma y talud. En ambientes modernos Lenticulina es libre epifaunal, vive en sustratos fangosos de plataforma externa y zona batial (Murray, 1991).

Hacia los niveles superiores de la sección, el decrecimiento en la frecuencia de los lagénidos se corresponde con el incremento de taxones de conchilla cónica pequeña de spirillínidos (Patellina subcretacea Cushman y Alexander) y de involutínidos (Trocholina infragranulata Noth) además de polimorfínidos tales como Globulina lacrima (Reuss), G. prisca (Reuss), Guttulina sp. y Pyrulina cylindroides (Roemer), lo cual estaría indicando una tendencia general a la somerización. A partir 
de la muestra $\mathrm{CH}$ 152, se verifica el pase a la suprayacente Formación Huitrín, con niveles caracterizados por la dominancia casi exclusiva de Patellina subcretacea y Guttulina sp. Asimismo, en cortes delgados se han observado valvas lisas de ostrácodos (Paracypris? sp.) de filiación marina.

El género Guttulina es considerado un indicador de condiciones marinas someras por Brouwer (1969); asimismo la abundancia de polimorfínidos, de hábito infaunal, ha sido considerada como indicadora de condiciones de fondo bien oxigenado (Bartolini et al., 1992). Por su parte, Koutsoukos y Hart (1990) reunieron a los polimorfínidos cretácicos de la cuenca de Sergipe, Brasil, en el submorfogrupo $\mathrm{CH}-\mathrm{B} .1$, con conchillas globulares u ovoidales, considerados formas móviles epi o infaunales, con hábitos detritívoros, viviendo indistintamente en sustratos calcáreos o silíceos de grano fino desde ambientes neríticos hasta batiales.

En ambientes modernos, Patellina Williamson es un taxón herbívoro epifaunal, prefiriendo sustratos duros, aguas templado frías y profundidades no mayores a 100 m (Murray, 1991). Por su parte, Berthold (1976) consideró a Patellina corrugata Williamson una forma móvil fital.

Como se ha señalado más arriba, en el perfil estudiado se observan intercalaciones calcáreas pelítico-arenosas con estructuras tractivas, atribuidas a esporádicos episodios de mayor energía producto de eventos de tormenta. Los mismos estarían representados principalmente en la muestra $\mathrm{CH} 6$ la cual es portadora prácticamente de la totalidad de los ostrácodos recuperados y de foraminíferos libres aglutinantes de material grueso (Sculptobaculites sp.) y de hábito adherido representados por Ammovertella? sp., Tolypammina? sp. y Bullopora laevis (Sollas). Sturrock y Murray (1981) señalaron que los foraminíferos aglutinados adheridos se ubican en ambientes someros de alta energía, aunque también pueden habitar ambientes más profundos tomando como sustratos a vegetales o animales. McLachlan et al. (1976a), al referirise a las microfaunas del Valanginiano superior de Sudáfrica, asocian la presencia de foraminíferos adheridos a un sustrato rocoso en un ambiente deposicional marino somero de aguas agitadas.

Los ostrácodos presentes, si bien de tamaño pequeño a mediano, poseen caparazones robustos, fuertemente calcificados, variablemente ornamentados y con charnelas bien desarrolladas, por ejemplo entomodonta en Majungaella pavta Ballent, Ronchi y Whatley y schizodonta en las especies de Sondagella Dingle. Valicenti y Stephens (1984) asociaron el desarrollo del prominente tubérculo ocular de Sondagella colchesterensis Valicenti y Stephens con un ambiente marino de plataforma de aguas poco profundas y con abundancia de alimentos. Se infiere entonces que estos elementos, propios de ambientes más someros, han sido transportados hasta la posición en la que se los encuentra. Asimismo, y a modo de confirmación, la presencia en la especie más abundante, $S$. colchesterensis, de una población formada casi íntegramente por caparazones adultos, indicaría un ambiente de depositación con cierta energía y con transporte posdeposicional. En el análisis comparado con las tipificaciones para modelos recientes de Whatley (1983), la estructura poblacional de esta especie es equiparable al tipo $B$, representada por una selección de tamaños con presencia de formas adultas y mudas mayores, faltando las mudas menores. Por último se menciona que tramos dominados por tormentas son frecuentes en los Miembros Pilmatué y Agua de la Mula (Leanza y Hugo, 2001).

En cuanto a la edad obtenida mediante los microfósiles bentónicos, se menciona que los foraminíferos son en su mayor parte formas longevas dentro del Cretácico Inferior, mientras que entre los ostrácodos hay formas acotadas al Valanginiano-Hauteriviano.

Los niveles basales portadores de Epistomina loncochensis Ballent contienen ammonites de la Zona de Pseudofavrella angulatiformis del Valanginiano tardío o son algo más antiguos y están asociados a la Zona de Olcostephanus (O.) atherstoni, correspondiente a la parte alta del Valanginiano temprano (cf. Sagasti y Ballent, 2002).

En el área del sur de la Provincia de Mendoza y norte del Neuquén, Simeoni y Musacchio (1996) ubicaron la primera aparición de Astacolus ambanjabensis Espitalié y Sigal, asociado al ostrácodo Sondagella colchesterensis Valicenti y Stephens y al cocolito Eiffelithus windii Applegate y Bergen en niveles del Valanginiano temprano.

Próximo al techo del perfil, se registra Lenticulina collignoni Espitalié y Sigal. La especie está representada en el Miembro Agua de la Mula en Bajada del Agrio (Provincia del Neuquén) nominando la Asociación de Planularia crepidularis-Lenticulina collignoni (Simeoni, 2000) del Hauteriviano tardío. 
En los sedimentos marinos regresivos de la base de la Formación Huitrín, Simeoni (2000) reconoció la 'fáunula' de Hergotella asignada al Barremiano que, en este caso, corresponde a los niveles del techo del perfil con abundancia de conchillas pequeñas cónicas de Patellina sub- cretacea Cushman y Alexander y polimorfínidos.

En cuanto a los nanofosiles recuperados, como se mencionó anteriormente, acotan la edad al Valanginiano superior-Hauteriviano superior, y se incluyen en las Biozonas CC4A y CC4B de Bown y Concheyro (2004).

\section{CONCLUSIONES}

En el presente trabajo se caracteriza micropaleontológicamente a la Formación Agrio en la sección Cuesta del Chihuido, en el sur de la Provincia de Mendoza, Argentina. Se han reconocido 37 especies de nanofósiles calcáreos, 34 de foraminíferos y seis de ostrácodos.

La secuencia, con un espesor de 205 m, se compone de pelitas negras, margas y calizas micríticas depositadas en un ambiente de baja energía y aguas relativamente profundas, localizado por debajo del nivel de olas de tormenta, en una zona de rampa marina abierta.

Bioestratigráficamente, y en conjunción con los amonites asociados, se han reconocido seis bioeventos de nanofósiles calcáreos, acotando la edad de la Formación Agrio en esta localidad al Valanginiano superior-Hauteriviano superior (Biozonas CC4A y CC4B). La FO de Eiffellithus striatus, la FO de Nannoconus circularis y de nanocónidos de canal ancho y la LO de Eiffellithus windii permiten asignar el Miembro Pilmatué al Valanginiano superior-Hauteriviano inferior. Por su parte, la presencia conjunta de Nannoconus bucheri y Lithraphidites bollii, la LO de Cruciellipsis cuvillieri y la LO de Eiffellithus striatus permiten asignar el Miembro Agua de la Mula al Hauteriviano superior.

En la base de la sección, el conspicuo banco portador de Epistomina loncochensis está relacionado con un episodio transgresivo que condujo a condiciones altamente eutróficas en la columna de agua (sugerido por la abundancia de radiolarios) y fondos poco aireados y deficitarios en oxígeno. Continúan condiciones marinas normales, con aguas claras y fondos fangosos bien oxigenados, con nanofósiles y una variada microfauna de foraminíferos calcáreos Lagenina. Hacia el techo, la abundacia de polimorfínidos, Involutinina y Spirillinina refleja una tendencia a la somerización. Esporádicos niveles calcáreos pelítico-arenosos atribuidos a episodios de mayor energía son interpretados como eventos de tormentas y son portadores de microfauna alóctona de foraminíferos libres aglutinantes de material grueso, adheridos, de pared aglutinada y calcárea y ostrácodos cytheroideos robustos y bien calcificados, predominando caparazones adultos que indican una selección de tamaños.

\section{AGRADECIMIENTOS}

Las autoras agradecen a la Dra. B. AguirreUrreta (Universidad de Buenos Aires) la determinación de los amonites hallados en el perfil y sus valiosos comentarios; a la licenciada G. Angelozzi (Consultora Bioestratigráfica, GEMA $\mathrm{SRL}$ ) por la importante discusión acerca de la presencia de algunos nanofósiles biomarcadores en la Cuenca Neuquina. Este trabajo contó con financiación del Consejo Nacional de Investigaciones Científicas y Técnicas (PIP 5613) y del proyecto PICT 14143/03. 


\section{APÉNDICE SISTEMÁTICO 1}

\section{LISTADO DE ESPECIES RECONOCIDAS}

Nanofósiles calcáreos (ordenados alfabéticamente)

Braarudosphaera bigelowi (Gran y Braarud) Deflandre, 1947.

Calculites suturus Bown y Concheyro, 2004.

Cretarhabdus conicus Bramlette y Martini, 1964.

Cruciellipsis cuvillieri (Manivit) Thierstein, 1971 emend. Wind y Cepek, 1979.

Cyclagelosphaera margerelii Noël, 1965.

Diazomatolithus lehmanii Nöel, 1965. Lám. 1, Fig. J.

Eiffellithus striatus (Black, 1971) Applegate y Bergen, 1989. Lám. 1, Figs. G, H.

Eiffellithus windii Applegate y Bergen, 1989. Lám. 1, Figs. E, F.

Ellispsagelosphaera britannica (Stradner, 1963) Reinhardt, 1964.

Ellispsagelosphaera ovata (Bukry, 1969) Black, 1973.

Haqius circumradiatus (Stover, 1966) Roth, 1978. Helenea chiasta Worsley, 1971.

Lithraphidites bollii (Thierstein, 1971) Thierstein, 1973. Lám. 1, Fig. D.

Lithraphidites carniolensis Deflandre, 1963.

Manivitella pemmatoidea (Deflandre) Thierstein, 1971, emend. Black, 1973.

Micrantholithus hoschulzii (Reinhardt, 1966);

Thierstein, 1971. Lám. 1, Fig. K.

Micrantholithus obtusus Stradner, 1963.
Nannoconus bucheri Brönnimann, 1955. Lám. 1, Fig. P.

Nannoconus circularis Deres y Achéritéguy, 1980. Nannoconus kamptneri Brönnimann, 1955. Lám. 1,

Fig. $\mathrm{O}$.

Nannoconus quadricanalis Bown y Concheyro, 2004.

Nannoconus sp.

Nannoconus steinmannii Kamptner, 1931. Lám. 1, Fig. $\mathrm{M}, \mathrm{N}$.

Retacapsa surirella (Deflandre y Fert, 1954); Grün in Grün y Allemann, 1975. Lám. 1, Fig. I.

Retecapsa crenulata (Bramlette y Martini, 1964); Grün in Grün y Allemann, 1975.

Rhagodiscus asper (Stradner) Reinhardt, 1967.

Staurolithites sp.

Stradnerlithus comptus Black, 1971.

Stradnerlithus silvaradius (Filewicz, Wise y Wind, in Wise y Wind, 1977); Rahman y Roth, 1991.

Tranolithus phacelosus Stover, 1966.

Watznaueria barnesae (Black y Barnes, 1959); Perch-Nielsen, 1968. Lám. 1, Fig. A.

Watznaueria biporta Bukry, 1969. Lám. 1, Fig. B.

Watznaueria fossacincta (Black, 1971); Bown y Cooper, 1989. Lám. 1, Fig. C

Watznaueria ovata Bukry, 1969.

Zeugrhabdotus embergeri (Noël, 1958) PerchNielsen, 1984. Lám. 1, Fig. L.

Zeugrhabdotus erectus (Deflandre in Deflandre y Fert, 1954); Reinhardt, 1965.

Zeughrabdotus sp.

Foraminíferos y ostrácodos

Orden Foraminiferida Eichwald, 1830

Suborden Textulariina Delage y Hérouad, 1896

Ammovertella? sp.

Lám. 2, Fig. A

Observaciones: ejemplar de hábito adherente, de pared finamente aglutinada, compuesta por una cámara hemisférica y tubular dispuesta en zigzag, que aumenta gradualmente en alto y ancho a medida que va creciendo. Abertura al final del tubo.
Valanginiano superior, muestra CH6. MLP-Mi 1461, ilustrado: $L=0,500$.

Tolypammina? sp. Lám. 2, Figs. B, C

Observaciones: dos ejemplares adherentes, finamente aglutinados, formados por una cámara 
tubular de crecimiento irregular; uno de ellos (MLPMi 1463) está adherido a otro foraminífero aglutinado cerca de la abertura del mismo; Fig. C). Valanginiano superior, muestra CH6. MLP-Mi 1462, 1463. Ilustrado MLP-Mi 1462 L=aprox. $1 \mathrm{~mm}$.

\section{Sculptobaculites sp.}

Lám. 2, Figs. D, E

Observaciones: conchilla planospiral involuta o con tendencia a evoluta, casi invariablemente deformada y comprimida en sección, de tamaño mediano a grande (entre $0,5 \mathrm{~mm}$ y algo más de $1,5 \mathrm{~mm}$ de diámetro) y que aglutina material arenoso grueso el cual enmascara en gran medida la morfología externa. Última vuelta con 8-9 cámaras separadas por suturas radiales, periferia lobulada más destacada en la parte final de la conchilla y área umbilical muy amplia. Recuerda fuertemente a $S$. goodlandensis (Cushman y Alexander) de McMillan (2003, p. 89, Figs. 31 A-B) del Valanginiano de la Cuenca de Algoa en Sudáfrica. Asimismo, es muy similar a las formas completamente enroscadas de Haphophragmoides sp. 2 de Masiuk y Viña, 1987 (p. 296, Lám. 3, Figs. 6-7) del Hauteriviano inferior del subsuelo del Chubut. Valanginiano superior, nivel CH6. MLP-Mi 1464-1468. Ilustrados MLP-Mi 1464, $\mathrm{DM}=1,40 ;$ MLP-Mi 1465, L=1,80.

Trochammina depressa Lozo, 1944 Lám. 2, Fig. F

Observaciones: Albiano, Texas (Lozo, 1944; Loeblich y Tappan, 1949); Valanginiano superior, noroeste de Alemania (Bartenstein y Brand, 1951); Aptiano-Albiano, Australia (Haig, 1980); rara en el Barremiano del Sitio 263, océano Índico, oeste de Australia (Holbourn y Kamisnki, 1997); Argentina: Hauteriviano-Barremiano, Provincia de Santa Cruz (Bertels, 1990) y Hauteriviano, Provincia del Neuquén (Simeoni, 1988). Valanginiano superior, muestra CH6. MLP-Mi 1469, 1470, 1471. Ilustrado MLP-Mi 1469, $\mathrm{DM}=$ 0, 640, Dm= 0, 510.

Marssonella subtrochus Bartenstein, 1962 Lám. 2, Fig. G

Observaciones: Cretácico Inferior, noroeste de Europa, Trinidad (Bartensteinetal., 1966); Valanginianosuperior, Sudáfrica (McLachlan et al., 1976b). Valanginiano superior, muestra CH6. MLP-Mi 1472, ilustrado L=1,30.

Suborden Involutinina Hohenegger y Piller, 1977

Trocholina infragranulata Noth, 1951

Lám. 2, Figs. $\mathrm{H}, \mathrm{I}$, J

Observaciones: vuelta final y relleno umbilical al mismo nivel, constituyen, según Guillaume (1963) una de las características diagnósticas de la especie, que la diferencian de otras especies típicas valanginianas, tales Trocholina valdensis (Reichel) y Trocholina molesta Gorbatchik. Hauteriviano, Austria; Valanginiano, Jura francés; Valanginiano superior, Sudáfrica (McLachlan et al., 1976a). Hauteriviano superior, muestras $\mathrm{CH} 127,142,154$. MLP-Mi 1524-1528. Ilustrados, MLP-Mi 1524, DM= $0,230, h=0,180 ;$ MLP-Mi 1528, $D=0,280, h=0$, 150. Tres ejemplares (MLP-Mi 1525-1527) DM entre 0,21 y $0,31 \mathrm{~mm}$, D/h entre 1,3 y algo menos de 2).

Suborden Spirillinina Hohenegger y Piller, 1975

Patellina subcretacea Cushman y Alexander, 1930

Lám. 2, Figs. K, L, M

Observaciones: Valanginiano-Aptiano, centro oeste de Europa; Valanginiano superior, Sudáfrica; Aptiano-Albiano, sitios profundos del océano Índico al noroeste de Australia (Holbourn y Kaminski, 1997). En Argentina, Hauteriviano, Cuenca Neuquina (Musacchio, 1979). Hauteriviano superior- 
Barremiano inferior, muestras $\mathrm{CH} 142,152,153$, 154, 157. MLP-Mi 1529-1534. Ilustrados MLP-Mi
1529, $\mathrm{DM}=0,200, \mathrm{H}=0,100 ; \mathrm{MLP}-\mathrm{Mi} 1530, \mathrm{D}=0$, 230, $\mathrm{H}=0,150$; MLP-Mi 1531, $\mathrm{DM}=0,280, \mathrm{H}=0,140$.

Suborden Lagenina Delage y Hérouard, 1896

\section{Pyramidulina sp. 1} Lám. 2, Fig. N

Observaciones: ejemplar megalosférico de cuatro cámaras subredondeadas, que crecen gradualmente en alto y ancho a medida que se agregan, separadas por suturas horizontales y muy deprimidas. Ornamentación constituida por diez costillas que recorren longitudinalmente la conchilla, continuas a través de las suturas y que llegan hasta la abertura, que es terminal y redondeada, al final de un pequeño cuello. Hauteriviano superior, muestra $\mathrm{CH} 142$. MLP-Mi 1473, ilustrado $\mathrm{L}=0,460$.

\section{Pyramidulina sp. 2}

Lám. 2, Fig. $\mathrm{O}$

Observaciones: fragmento con las dos últimas cámaras de una conchilla robusta, con suturas apenas insinuadas y ornamentada por once costillas filosas, continuas a través de las suturas. Abertura terminal, redondeada, con un pequeño aunque notorio cuello. Valanginiano superior, muestra CH10. MLP-Mi 1474, L= 0,345 (fragmento).

\section{Lenticulina collignoni Espitalié y Sigal, 1963} Lám. 2, Fig. $P$

Observaciones: Jurásico Superior-Valanginiano inferior, Cuenca de Majunga, Madagascar; Hauteriviano, Cuenca Neuquina (Musacchio, 1979; Masiuk y Viña, 1986; Simeoni, 1988). Hauteriviano superior, muestra $\mathrm{CH} 147$. $\mathrm{L}=0,400$ (ejemplar perdido después de ser fotografiado).

Lenticulina sp. Lám. 2, Fig. Q

Observaciones: conchilla planoespiral involuta, biumbilicada, con ocho cámaras en la última vuelta, separadas por suturas curvadas, a nivel entre las primera cámaras y ligeramente deprimidas entre las últimas. Periferia aguda aunque no presenta carena. Recuerda Lenticulina circumcidanea (Berthelin) en Holbourn y Kaminski (1997, Lám. 31, fig. 8) del Neocomiano de sitios del Océano Índico, si bien ese material posee una delgada carena periférica y cara apertural más destacada. Hauteriviano superior, muestra CH142. MLP-Mi 1475, ilustrado $\mathrm{DM}=0,370, \mathrm{Dm}=0,260, \mathrm{E}=0,150$.

Marginulinopsis bettenstaedti Bartenstein

y Brand, 1951

Lám. 2, Figs. R, S

Observaciones: Kimmeridgiano-Albiano del Hemisferio Norte; Valanginiano-Hauteriviano, sitio 249, al sudoeste de Madagascar (Riegraf, 1989) y otros sitios profundos del océano Índico (Hauteriviano-Barremiano al oeste de Australia, Holbourn y Kaminski, 1997). En Argentina, Hauteriviano, Cuenca Austral (Malumián y Masiuk, 1975; Malumián y Náñez, 1983; Bertels, 1990). Hauteriviano superior, muestra CH142. MLP-Mi 14761479. Ilustrados MLP-Mi 1476, $L=0,530, a=0,280$; MLP-Mi 1477, L=0,460, a= 0,200.

\section{Pravoslavlevia tsaramandrosoensis (Espitalié y Sigal, 1963) Lám. 2, Fig. T}

Observaciones: Cristellaria italica (Defrance) del Neocomiano de la Cuenca de Gingin (Australia) podría ser coespecífico con la presente (cf. Chapman, 1917, lám. 9, Fig. 78). McMillan, 2003 (p. 192) incluye los especímenes de Argentina en $P$. frankei (Ten Dam). Kimmeridgiano-Valanginiano inferior, Cuenca de Majunga, Madagascar; ValanginianoHauteriviano, Sudáfrica (McLachlan et al., 1976a). En Argentina, Valanginiano, Cuenca Austral (Malumián y Masiuk, 1975; Kielbowicz et al., 1983; Malumián y Náñez, 1983; Masiuk y Viña, 1986); Hauteriviano, Cuenca Neuquina (Musacchio, 1979). Hauteriviano superior, muestra $\mathrm{CH} 142)$. MLP-Mi 1480 , ilustrado, $L=0,350, a=0,150, E=0,080$. 
Astacolus ambanjabensis Espitalié y Sigal,
1963 Lám. 2, Fig. U

Observaciones: Astacolus sp. A de McLachlan et al., 1976b (p. 353, Figs.12, 1-4) del Valanginiano superior de Sudáfrica probablemente sea coespecífica. Jurásico Superior, Cuenca de Majunga, Madagascar; Valanginiano tardío (como Astacolus sp. 1, Simeoni, 1985) y Hauteriviano, Cuenca Neuquina (Musacchio, 1979, Masiuk y Viña, 1986 y Cuenca Austral (Kielbowicz et al., 1983; Bertels, 1990). Valanginiano superior-Hauteriviano inferior, muestra CH6, 8, 10, 13, 15. MLP-Mi 1481-1486. llustrados, MLP-Mi $1483 \mathrm{~L}=0,680$, D espira $=0,5$.

\section{Astacolus beerae Brenner y McMillan (in McLachlan et al., 1976b) \\ Lám. 2, Fig. V}

Observaciones: Vaginulina sp. de Kielbowicz et al., 1983 (Lám. 4, Fig. 12) del Valanginiano de Cuenca Austral es externamente similar, aunque posee ocho costillas menos filosas y la cara apertural más alargada. Restringida al Valanginiano superior de Sudáfrica. Valanginiano superior, muestras $\mathrm{CH} 13$. MLP-Mi 1487-1489. Ilustrado, MLP-Mi 1487 L=0, $480, A=0,210, E=0,150$.

Astacolus calliopsis (Reuss, 1863)

Lám. 2, Fig. W

Observaciones: el ejemplar ilustrado por Musacchio (1979 en Lám. 4, fig. 22), así como el ilustrado por Simeoni (1988, Lám. 1, Fig. 16) de la Cuenca Neuquina, en ambos casos identificados como Astacolus schloenbachi (Reuss) se comparan muy bien con el descrito en este trabajo. ValanginianoAptiano, sitios en el océano Índico (Holbourn y Kaminski, 1997); Valanginiano tardío, Sudáfrica (McLachlan et al., 1976a); Valanginiano tardíoHauteriviano tardío, Sudáfrica (McMillan, 2003). En Argentina, Hauteriviano, Cuenca Neuquina (Musacchio, 1979). Valanginiano superior, muestra $\mathrm{CH} 13$. MLP-Mi 1490 ilustrado, $L=0,570, A=0,250, E=0,20$.

Marginulina schloenbachi (Reuss, 1863) Lám. 3, Fig. A

Observaciones: Valanginiano-Aptiano, sitios en el océano Indico (Holbourn y Kaminski, 1997); Valanginiano tardío de Sudáfrica (McLachlan et al., 1976b); Valanginiano tardío-Hauteriviano tardío, Sudáfrica (McMillan, 2003.) En Argentina, Hauteriviano, Cuenca Austral (Bertels, 1990). Hauteriviano superior, muestra $\mathrm{CH} 142$. MLP-Mi 1491 ilustrado, $L=0,440, A=0,150$.

$$
\begin{aligned}
& \text { Marginulina? sp. } \\
& \text { Lám. 3, Fig. B }
\end{aligned}
$$

Observaciones: se trata de fragmentos uniseriales asignados con dudas a Marginulina ornamentados por costillas finas continuas a través de las suturas que son fuertemente deprimidas. Formas similares están ilustradas como M. caelata Loeblich y Tappan por Riegraf (1989) del Caloviano-Valanginiano del sitio 249 del sudoeste de Madagascar. Hauteriviano tardío, muestra CH142. MLP-Mi 1492 ilustrado, L= 0,425 .

$$
\begin{aligned}
& \text { Vaginulinopsis matutina (d'Orbigny, 1849) in } \\
& \text { Macfadyen, } 1936 \\
& \text { Lám. 3, Figs. C, D }
\end{aligned}
$$

Observaciones: Lías de Europa; Cretácico Inferior, Trinidad (Bartenstein et al., 1957) y Sudáfrica (McLachlan et al., 1976b). Hauteriviano superior, muestra CH142. MLP-Mi 1493-1494. Ilustrados, MLP-Mi $1493 \mathrm{~L}=0,550$, D espira= 0, 300, D porción uniserial= 0, 210; MLP-Mi $1494 \mathrm{~L}=0,510$, D espira= 0,175, D porción uniserial $=0,150$.

\section{Vaginulinopsis sp. Lám. 3, Fig. E}

Observaciones: escasos ejemplares con afinidades externas con $V$. matutina (d'Orbigny) del mismo nivel, diferenciándose porque posee la conchilla con periferia ventral marcadamente redondeada y las suturas no son deprimidas, sino a nivel de la superficie. Hauteriviano superior, muestra $\mathrm{CH} 142$. Ilustrado, MLP-Mi $1495 \mathrm{~L}=0,590$, D espira= 0, 200, D porción uniserial $=0,220, E$ espira $=0,180$.

Citharina intumescens var. culter (Fursenko y Polenova, 1950 ) sensu Espitalié y Sigal, 1963 Lám. 3, Fig. J

Observaciones: Jurásico Superior (Volgiano inferior) 
de Rusia y Berriasiano-Valanginiano inferior, Cuenca de Majunga (Madagascar). Valanginiano superior, muestras CH13, 15. MLP-Mi 1496-1497. llustrado MLP-Mi $1496 \mathrm{~L}=0,760, E=0,100$.

\section{Citharina sparsicostata (Reuss) sensu Bartenstein y Brand, 1951 Lám. 3, Fig. F}

Observaciones: los ejemplares de esta especie del Cretácico Inferior de Cuenca Austral ilustrados por Kielbowicz et al. (1983, Lám. 1, fig. 10) y por Malumián y Náñez (1983, Lám. 1, Figs. 15-16), son actualmente referidos a $C$. austroafricana McMillan, 2003 (p. 155) ya que son más alargados, las cámaras son menos inclinadas hacia la parte inicial de la conchilla y las costillas son más profusas. Valanginiano-Albiano, Europa; Berriasiano-Valanginiano inferior, Cuenca de Majunga (Madagascar). Valanginiano superior-Hauteriviano superior, muestra CH13, 142. MLP-Mi 1498, 1499. Ilustrado MLP-Mi $1498 \mathrm{~L}=0,480, A=0,360$.

\section{Citharina cristellarioides (Reuss) sensu Bartenstein y Brand, 1951 \\ Lám. 3, Fig. G}

Observaciones: Valanginiano superior, noroeste de Alemania; Berriasiano-Valanginiano, Madagascar (Espitalié y Sigal, 1963). Hauteriviano inferior en diferentes secciones de la Cuenca Neuquina (Musacchio, 1979, Simeoni, 1985). Valanginiano superior, muestra $\mathrm{CH} 6,13$. MLP-Mi 1500, fragmento $L=0,850$.

Planularia complanata (Reuss, 1845) Lám. 3, Figs. $\mathrm{H}, \mathrm{I}$

Observaciones: Hauteriviano, Aptiano y Albiano de Europa; Albiano (Sitio 259), ValanginianoBarremiano (Sitios 249 y 263), Berriasiano-Albiano (Sitio 763 ) y Valanginiano-Barremiano (Sitio 766), todos del fondo del Océano Índico, noroeste de Australia (Holbourn y Kaminski, 1997). En Argentina, Hauteriviano, Cuenca Austral (Bertels, 1990), actualmente referida a $P$. formosa McMillan, 2003 (p. 187). Hauteriviano superior, muestra $\mathrm{CH} 142$. MLP-Mi 1501, 1502. Ilustrados MLP-Mi $1501 \mathrm{~L}=0$, $310, A=0,200$; MLP-Mi $1502 L=0,350, A=0,175$.
Planularia crepidularis Roemer, 1842 Lám. 3, Figs. K, L

Observaciones: Valanginiano- Barremiano inferior, Europa; Barremiano medio-Aptiano, Trinidad; Valanginiano-Hauteriviano, Cuenca de Majunga (Madagascar) y Valanginiano superior de cuencas sudafricanas; Valanginiano-Barremiano, sitios de fondo del Océano Indico al este de África (Riegraf, 1989) y del noroeste de Australia (Holbourn y Kamisnki, 1997). En Argentina, Hauteriviano superior, Cuenca Neuquina (Simeoni, 1988) y Valanginiano-Hauteriviano, Cuenca Austral (Malumián y Masiuk, 1975; Malumián y Náñez, 1983). Valanginiano superior, muestra $\mathrm{CH} 13,15$. MLP-Mi 1503-1507. Ilustrados MLP-Mi 1503 fragmento $L=$ $0,540, A=0,320 ;$ MLP-Mi $1504 L=0,700, A=0,340$

Psilocitharella arguta (Reuss, 1860) Lám. 4, Fig. A

Observaciones: Psilocitharella kochii (Roemer) ilustrada por Simeoni (1988, Lám. 2, Fig.1) de la sección superior de la Formación Agrio es muy similar salvo que tiene el borde dorsal ligeramente curvo, sobre todo en la porción inicial. Por su parte, P. gaultina (Berthelin) de Simeoni (1988, Lám. 2, Fig. 2) presente en los mismos niveles señalados, posee similar aspecto pero está ornamentada por costillas muy delicadas. Valanginiano-Barremiano, Europa; Aptiano (Sitio 260) y Valanginiano superiorHauteriviano (sitio 766) al noroeste de Australia (Holbourn y Kaminski, 1997); Hauteriviano superior, Sudáfrica (McMillan, 2003). Hauteriviano, Neuquén Musacchio (1979). Hauteriviano superior, muestra CH142. MLP-Mi 1508 ilustrado $L=0,770 ; E=0,090$.

\section{Reussoolina? sp. Lám. 3, Fig. M}

Observaciones: conchilla unilocular pequeña, de forma ovoidal, ligeramente comprimida en vista dorsal debido a que posee una sutil carena periférica en ambos márgenes. Superficie lisa. Abertura radiada algo protruyente. Se mantiene la nomenclatura abierta debido a que en el género Reussoolina la abertura es redondeada, rodeada de surcos radiales, en vez de radiada como en el ejemplar estudiado. Hauteriviano superior, muestra $\mathrm{CH} 142$. MLP-Mi 1509 ilustrado $L=0,280 ; E=0,120$. 
Globulina lacrima (Reuss, 1845) Lám. 3, Fig. N

Observaciones: Cretácico, Hemisferio norte; sitios del Océano Índico: Valanginiano-Barremiano al sudoste de Madagascar y Valanginiano-Albiano al noroeste de Australia (Holbourn y Kaminski, 1997). Hauteriviano superior, muestra CH142. MLP-Mi 1510 ilustrado $L=0,320$.

\section{Globulina prisca (Reuss, 1863) Lám. 3, Fig. R}

Observaciones: Cretácico, hemisferio norte; Valanginiano superior, Sudáfrica (McLachlan et al., 1976a, b; McMillan, 2003) y Berriasiano- Albiano, sitios profundos del Océano Índico. Hauteriviano, Cuenca Neuquina (Masiuk y Viña, 1986) y Cuenca Austral (Malumián y Náñez, 1983). Hauteriviano superior, muestra CH142. MLP-Mi 1511 ilustrado $\mathrm{L}=0,330$.

\section{Guttulina sp.}

Lám. 3, Figs. O, P, Q

Observaciones: conchilla muy pequeña, globular aunque comprimida en sección apertural. Cámaras dispuestas en planos a $144^{\circ}$ entre sí, y separadas por suturas oblicuas y nítidas, variablemente deprimidas en los diferentes ejemplares. La cámara basal es algo sobresaliente y angulosa con ápice basal y las otras cámaras son envolventes desde la base. Abertura terminal, radiada. El material estudiado, aunque abundante, presenta un regular estado de conservación, con muchos ejemplares aplastados y deformados, lo que sumado a su pequeño tamaño, dificulta su descripción y asignación específica. Guttulina sp. 1 de Musacchio (1979, Lám. 5, Fig. 11) del Hauteriviano de la Cuenca Neuquina es similar externamente aunque con conchilla algo más elongada. Hauteriviano superiorBarremiano inferior, muestras $\mathrm{CH} 142,153,154$, 157. Ilustrado MLP-Mi 1512, L=0,280; MLP-Mi 1513, $L=0,260$.
Pyrulina cylindroides (Roemer, 1838)

Lám. 3, Fig. S

Observaciones: Cretácico, aunque Riegraf y Luterbacher (1989) extienden su biocrón hasta el Reciente; Valanginiano superior y Hauteriviano inferior tardío, Sudáfrica (McLachlan et al., 1976a y McMillan, 2003, respectivamente); ValanginianoAptiano, sitios profundos del Atlántico norte (Riegraf y Luterbacher, 1989) y Valanginiano, Aptiano y Albiano, sitios profundos del Océano Índico, noroeste de Australia (Holbourn y Kaminski, 1997). Hauteriviano, Cuenca Neuquina (Musacchio, 1979). Hauteriviano superior, muestra $\mathrm{CH} 142$. MLP-Mi 1517. Ilustrado $L=0,330$.

Bullopora laevis (Sollas, 1877)

Lám. 4, Figs. B, C

Observaciones: ubicada originariamente en el género Webbina d'Orbigny por su aparente pared calcárea porcelanácea. Se diferencia de otra especie cretácica, Bullopora tuberculata (Sollas), porque ésta posee la superficie cubierta por pequeños tubérculos. Bullopora sp. de Kielbowicz et al., 1983 (Lám. 5, Fig. 2) del Valanginiano de la Formación Springhill en el sector argentino de la Cuenca Austral, podría ser coespecífica con la presente. Cretácico Inferior inglés (Adams, 1962); Valanginiano medio-superior, noroeste de Alemania (Bartenstein y Brand, 1951); Cretácico Superior, Cuenca de Gingin (Chapman, 1917) y Albiano de Queensland (Haig, 1982), ambas en Australia; Valanginiano-Hauteriviano, Sudáfrica (McMillan, 2003). Valanginiano superior, muestra CH6. MLPMi 1518-1520. Ilustrado MLP-Mi 1518 L= 0,600.

Webbinella? sp. Lám. 4, Fig. D

Observaciones: conchilla de hábito adherente, probablemente perteneciente a Webbinella con una porción inicial con cámaras con disposición polimorfínido y la última cámara circundante fija parcialmente disuelta, dejando una marca que 
reconstruye la periferia de la misma. No es fácil identificar a los foraminíferos que viven adheridos a un sustrato duro ya que su morfología está com- pletamente condicionada por la forma de aquél. Hauteriviano superior, muestra $\mathrm{CH} 147$. Ilustrado MLP-Mi $1521 \mathrm{~L}$ aproximadamente $1 \mathrm{~mm}$.

Suborden Robertinina Loeblich y Tappan, 1984

Epistomina loncochensis Ballent (in Sagasti y Ballent, 2002)

Lám. 4, Fig. E

Observaciones: frecuente en los niveles basales de la Formación Agrio (Valanginiano inferior alto, Valanginiano superior) en diferentes secciones del sector septentrional de la Cuenca Neuquina (cf.
Sagasti y Ballent, 2002). Valanginiano superior, muestra $\mathrm{CH} 3$, 4, y fragmentos en $\mathrm{CH}$ 144. MLP-Mi 1522, 1523. Ilustrado MLP-MI $1522 \mathrm{DM}=0,680$, $\mathrm{Dm}=0,630, \mathrm{E}=0,400, \mathrm{~N}$. cám $=10$ (incompleto).

Clase Ostracoda Latreille, 1806

Familia Cytherideidae Sars, 1925

Rostrocytheridea sp. nov.?

Lám. 4, Fig. $\mathrm{F}$

Observaciones: especie mediana con grueso reticulado dispuesto paralelo a la periferia de la valva. Hauteriviano superior, muestra $\mathrm{CH} 147$. MLPMi $1424 L=0,575, A=0,305, E=0,250$

\section{Mandelstamia? sp.} Lám. 4, Fig. G

Observaciones: caparazón de tamaño mediano, subtriangular elongado en vista lateral con la mayor altura en el tercio anterior y simétricamente acuminado posteriormente. Borde anterior ampliamente redondeado, más destacado ánteroventralmente. Borde dorsal, claramente descendente hacia atrás y con una cancavidad en la parte media. Bordes dorsal y ventral convergentes hacia la parte posterior. Ornamentación constituida por un reticulado concéntrico más grueso en el área central de la valva y dispuesto paralelo a la periferia. Recuerda Mandelstamia cf. 962 Grekoff de Brenner y Oertli, 1976 (p. 498, Lám. 5, Figs. 1-4) del Hauteriviano de Sudáfrica, aunque ésta es más comprimida en los márgenes del caparazón. Hauteriviano superior, muestra $\mathrm{CH}$ 147. Ilustrado MLP-Mi 1433, $L=0,600, A=0,250, a=0,200$.

Familia Cytheruridae G.W. Müller, 1894

Procytherura sp. nov.?

Lám. 4, Fig. H

Observaciones: especie pequeña, subrectangular elongada, con costilla ventro lateral bien definida y ornamentada por un delicado retículo dispuesto paralelo al borde ventral. Podría ser coespecífica con Procytherura cf. maculata Brenner y Oertli de Simeoni y Musacchio, 1996 (p. 1412, Lám. 2) del Valanginiano tardío. Valanginiano superior, muestra CH6, 142. MLP-Mi 1435L=0, 410, A=0, 200, a= 0, 180. 
Familia Progonocytheridae Silvester-Bradley, 1948

Majungaella pavta Ballent, Ronchi y Whatley, 1998

Lám. 4, Fig. I

Observaciones: presente en el Titoniano superiorBerriasiano, Formación Loma Montosa, subsuelo del sector oriental de la Cuenca Neuquina (Ballent et al., 1998); Valanginiano inferior de la localidad
Barranca de los Loros, Provincia del Neuquén (Schwarz, 2003). Valanginiano superior, muestra CH13. MLP-Mi 1443, 1444. Ilustrado MLP-Mi 1443 $L=0,770, A=0,390, a=0,390$.

Familia Schizocytheridae Howe, 1961

\author{
Sondagella colchesterensis Valicenti y \\ Stephens, 1984 \\ Lám. 4, Fig. J
}

Observaciones: esta especie es la más abundante en las muestras estudiadas, recuperándose quinientos caparazones y cuatro valvas. Aparentemente restringida al Hauteriviano superior en la Cuenca de Algoa en Sudáfrica. Valanginiano superior, muestra GH6, 8, 10, 13, 142. MLP-Mi 1446-1460. llustrados MLP-Mi $1446 \mathrm{~L}=0,530, A=0,290, a=0,200$.

Sondagella theloides Dingle, 1969 Lám. 4, Fig. K

Observaciones: Hauteriviano, Cuenca Neuquina; Valanginiano superior- Hauteriviano superior, Sudáfrica. Valanginiano superior, muestra $\mathrm{CH} 10$. MLP-Mi 1445, ilustrado $L=0,450, A=0,210, a=0,200$.

\section{REFERENCIAS}

Adams, C.G. 1962. Calcareous adherent Foraminifera from the British Jurassic and Cretaceous and the French Eocene. Palaeontology 5 (2): 149-170.

Aguirre-Urreta, M.B. 2001. Marine Upper Jurassic-Lower Cretaceous stratigraphy and biostratigraphy of the Aconcagua-Neuquén Basin, Argentina and Chile. Journal of Iberian Geology 27: 71-90.

Aguirre-Urreta, M.B.; Rawson, P. 1997. The ammonite sequence in the Agrio Formation (Lower Cretaceous), Neuquén Basin, Argentina. Geological Magazine 134 (4): 449-458.

Applegate, J.L.; Bergen, J.A. 1989. Cretaceous calcareous nannofossil bioestratigraphy of sediments recovered from Galacia margin, ODP. Leg. 103. Proceedings of the Ocean Drilling Project, Scientific Results 103: 293-326.

Ballent, S.C.; Ronchi, D. I.; Whatley, R. 1998. The ostracod genus Majungaella Grekoff in Argentina. Revista Geológica de Chile 25 (1): 45-55.

Bartenstein, H. 1962. Neue Foraminiferen aus Underkreide und Oberkeuper NW-Deutschland und der Schweiz. Senckenbergiana lethaea 43 (2): 135-149.

Bartenstein, H.; Brand, E. 1951. Mikropaläontologische
Untersuchungen zur Stratigraphie des nordwestdeutschen Valendis. Abhandlungen der Senckenbergischen Naturforschenden Gesellschaft 485: 239-336.

Bartenstein, H.; Bettenstaedt, F.; Bolli, H.M. 1957. Die Foraminiferen der Unterkreide von Trinidad, B.W.I. Erster Teil: Cuche-und Toco-Formation. Eclogae Geologica Helvetiae 50 (1): 5-67.

Bartenstein, H.; Bettenstaedt, F.; Bolli, H.M. 1966. Die Foraminiferen der Unterkreide von Trinidad, W.I. Zweter Teil: Maridale-Formation (Typlokalität). Eclogae Geologica Helvetiae 59 (1): 129-177.

Bartolini, A.; Nocchi, M.; Baldanza, A.; Parisi, G. 1992. Benthic life during the early Toarcian anoxic event in the Southwestern Tethyan Umbria-Marche Basin, Central Italy. Studies on Benthic Foraminifera BENTHOS '90, Sendai. Tokai University Press: 323338.

Bertels, A. 1990. Foraminíferos bentónicos y caracterización paleoambiental de la Formación Río Mayer (Hauteriviano-Barremiano) en Lago San Martín, República Argentina. Revista Española de Micropaleontología 22 (2): 239-293.

Berthold, W.U. 1976. Test morphology and morphogenesis 
in Patellina corrugata Williamson, Foraminiferida. Journal of Foraminiferal Research 6 (3): 167-185.

Black, M. 1971. Coccoliths of the Speeton Clay and Sutterby Marl. Proceedings of the Yorkshire Geological Society 38 (3): 381-424.

Black, M. 1973. British Lower Cretaceous Coccoliths.I. Gault Clay (Part 2). Paleontological Society of London (Monograph) 127: 49-112.

Black, M.; Barnes, B. 1959. The structure of coccoliths from the English chalk. Geological Magazine 96 (5): 321-328.

Bown, P.; Cooper, M.K.E. 1989. New calcareous nannofossils from the Jurassic. Journal of Micropalaeontology 8 (1): 91-96.

Bown, P.R.; Young, J. 1998. Techniques in Calcareous Nannofossil Biostratigraphy. In Calcareous Nannofossil Biostratigraphy (Bown, P.; editor). British Micropaleontological Series, Chapman and Hall: 1628. Cambridge.

Bown, P.; Concheyro, A. 2004. Lower Cretaceous calcareous nannoplankton from the Neuquén Basin Argentina. Marine Micropaleontology 52 (1): 51-84.

Bramlette, M.N.; Martini, E. 1964. The great change in calcareous nannoplankton fossils between the Maestrichtian and Danian. Micropaleontology 10 (3): 291-322.

Brenner, P.; Oertli, H. 1976. Lower Cretaceous Ostracode (Valanginian to Hauterivian) from Sundays River Formation, Algoa Basin, South Africa. Centre Recherches Pau, Bulletin, Societé Nationale des Pétroles d'Aquitaine 10 (2): 471-533.

Brönnimann, P. 1955. Microfossils incertae sedis from the Upper Jurassic and Lower Cretaceous of Cuba. Micropaleontology 1 (1): 28-51.

Brouwer, J. 1969. Foraminiferal assemblages from the Lias of North-Western Europe. Verhandelingen der Koninklije Nederlandse Akademie Van wetenschappen, Afd. Natuurkunde 25 (4): 1-48.

Bukry, D. 1969. Upper Cretaceous coccoliths from Texas and Europe. University of Kansas Paleontology Contributions (51) (Protista 2): 1-75.

Burchette, T.P.; Wright, V.G. 1992. Carbonate ramp depositional systems. Sedimentary Geology 79 (1-4) 3-57.

Chapman, F. 1917. Monograph of the Foraminifera and Ostracoda of the Gingin Chalk. Palaeontological Contributions to the Geology of Western Australia. Series VI (11) Western Australia Geological Survey Bulletin (72), series 6 (11): 9-87.

Cushman, J.A.; Alexander, C.L. 1930. Some vaginulids and other Foraminifers from the Lower Cretaceous of Texas. Contributions from the Cushman Laboratory for Foraminiferal Research 6 (1): 1-10.

Deflandre, G. 1947. Braasrudosphaera nov. gen., type d'une famille nouvelle de coccolithophoridés actuels à éléments composites. Comptes Rendus Hebdomadaires des Seances de l'Academie des Sciences 225: 439-441. Paris.
Deflandre, G. 1963. Sur Microrhabdulidés, famille nouvelle de nannofossiles calcaires. Comptes Rendus (Hebdomadaires des Seances de l'Academie des Sciences 256: 3484-3486. Paris

Deflandre, G.; Fert, C. 1954. Observations sur les Coccolithophoridés actuels et fossils en microscopie ordinaire et électronique. Annales de Paléontologie 40 (2): 115-176.

Delage, Y.; Hérouard, E. 1896. Traité de Zoologie Concrète. Tome I. La cellule et les Protozaires: $589 \mathrm{p}$. Paris.

Deres, F.; Acheriteguy, J. 1980. Biostratigraphy of Nannoconids. Centre Recherches Exploration Production Elf-Aquitaine, Bulletin 4 (1): 1-53.

Dingle, R.V. 1969. Marine Neocomian Ostracoda from South Africa. Transactions of the Royal Society of South Africa 38 (2): 139-163.

Edwards, A. 1963. A preparation technique for calcareous nannoplankton. Micropaleontology 9 (1): 103-104.

Eichwald, C.E. von 1830. Zoologia specialis 2. Vilnae: D.E. Eichwaldus: 1-323.

d'Orbigny, A.D. 1849. Foraminifères. In Dictionnaire Universel d' Histoire Naturelle. Renard, Martinet and Cie. 5: 662-671. Paris

Espitalié, J.; Sigal, J. 1963. Contribution à l'étude des foraminifères (micropaléontologie-microstratigraphie) du Jurassique Supérieur et du Néocomien du bassin de Majunga (Madagascar). Annales Géologiques de Madagascar (32): 1-99.

Fursenko, A.V.; Polenova, E.N. 1950. Foraminifères du Volgien inférieur de la région d' Emba. Travaux Institut Géologie et Recherches Pétrole, n. s. 49: p. 71.

Grün, W. von; Allemann, F. 1975. The lower Cretaceous of Caravaca (Spain): Berriasian calcareous nannoplankton of the Miravetes section (Subbetic Zone, Prov. of Murcia). Eclogae Geologica Helvetica 68 (1): 147-211.

Guillaume, S. 1963. Les trocholines du Crétacé inférieur du Jura. Revue de Micropaléontologie 5 (4): 257-276.

Haig, D.W. 1980. Early Cretaceous Textulariine foraminiferids from Queensland. Palaeontographica (A) 87: 1-138.

Haig, D.W. 1982. Early Cretaceous Milioline and Rotaliine benthic foraminiferids from Queensland. Palaeontographica A 177: 1-88

Hohenegger, J.; Piller, W. 1975. Wandstrukturen und Grossgliederung der Foraminiferen. Sitzungsberichten der Österreichisch Akademie der Wissenschaften, Mathematisch-naturwissenschaftliche Klasse, Abteilung I 184 (1-5): 67-96.

Hohenegger, J.; Piller, W. 1977. Die Stellung der Involutinidae Bütschi und Spirillinidae Reuss im System der Foraminiferen. Neues Jahrbuch für Geologie und Paläontologie, Monatshefte 197 (7): 407-418.

Holbourn, A.E.; Kaminski, M.A. 1997. Lower Cretaceous deep-water benthic foraminifera of the Indian Ocean (a synthesis of DSDP and ODP material). Grzybowski 
Foundation, Special Publication (4): 172 p. Polonia.

Howe, H.V. 1961. Family Schizocytheridae. In Treatise on Invertebrate Paleontology. Part Q Arthropoda 3, p. 331 (Moore, R.; Pitrat, C.; editors). Geological Society of America and University Kansas Press: 442 p. Lawrence.

Kamptner, E. 1931. Nannoconus steinmanni nov. gen. nov. spec., ein merkwûrdiges gesteinsbildenses Mikrofossil aus dem jungeren Mesozoikum der Alpen. Paläontologische Zeitschrift 13: 288-298.

Kielbowicz, A.A.; Rionchi, D.I.; Stach, H.H. 1983. Foraminíferos y ostrácodos valanginianos de la Formación Springhill, Patagonia Austral. Revista de la Asociación Geológica Argentina 38 (3-4): 313-339.

Koutsoukos, E.A.M.; Hart, M.B. 1990. Cretaceous foraminiferal morphogroup distribution patterns, palaeocommunities and trophic structures: a case study from the Sergipe Basin, Brazil. Proceedings of the Royal Society of Edinburgh: Earth Sciences 81 (3): 221-246.

Latreille, P. A. 1806. Genera crustaceorum et insectorum. Amand Koening, Paris et Argentorani 1: 1-302.

Leanza, H.A.; Hugo, C.A. 2001. Hoja Geológica 3969-I Zapala, Provincia del Neuquén. Servicio Geológico Minero Argentino, Instituto de Geología y Recursos Minerales, Boletín (275): 133 p. (en CD ROM).

Legarreta. L.; Gulisano, C. 1989. Análisis estratigráfico secuencial de la Cuenca Neuquina (Triásico SuperiorTerciario Inferior), Argentina. In Cuencas Sedimentarias Argentinas (Chebli, G. A.; Spalletti, L. A; editores). Serie de Correlación Geológica 6: 221-243.

Legarreta, L.; Uliana, M.A. 1991. Jurassic-Cretaceous marine oscillations and geometry of back-arc basin fill, central Argentina Andes. In Sedimentation, tectonics and eustasy (McDonald, D.I.M.; editor). International Association of Sedimentologists, Special Publication (12): 429-450.

Loeblich, A.R. Jr.; Tappan, H. 1949. Foraminifera from the Walnut Formation (Lower Cretaceous) of northern Texas and southern Oklahoma. Journal of Paleontology 23 (3): 245-266.

Loeblich, A.R. Jr.; Tappan, H. 1984. Suprageneric classification of the Foraminiferida (Protozoa). Micropaleontology 30 (1): 1-70.

Loeblich, A. R. Jr.; Tappan, H. 1988. Foraminiferal genera and their classification. Van Nostrand Reinhold Company: 970 p. New York.

Lozo, F.E. Jr. 1944. Biostratigraphic relations of some North Texas Trinity and Fredericksburg (Comanchean) foraminifera. American Midland Naturalist 31: 513582.

Macfadyen, W. 1936. D'Orbigny's Lias Foraminifera. Journal Royal Microscopical Society 3: 147-153.

Malumián, N.; Masiuk, V. 1975. Foraminíferos de la Formación Pampa Rincón (Cretácico Inferior), Tierra del Fuego, Argentina. Revista Española de Micropaleontología 7 (3): 579-600.

Malumián, N.; Náñez, C. 1983. Foraminíferos de ambiente anóxico de la Formación Río Mayer (Cretácico Inferior) Provincia de Santa Cruz. Ameghiniana 20 (3-4): 367393.

Masiuk, V.; Viña, F. J. 1986. Estratigrafía de la Formación Agrio de la Cuenca Neuquina. Boletín de Informaciones Petroleras, Tercera Época (6) p. 2-38.

Masiuk, V.; Viña, F. J. 1987. Foraminíferos del Hauteriviano inferior del subsuelo de Chubut, Argentina. I: Aglutinados. Ameghiniana 24 (3-4): 89-298.

McLachlan, I.R.; McMillan, I.K.; Brenner, P.W. 1976a. Micropalaeontological study of the Cretaceous beds at Mbotyi and Mngazana, Transkei, South Africa. Transactions of the Geological Society of South Africa 79 (3): 321-340.

McLachlan, I.R.; Brenner, P.W.; McMillan, I.K. 1976b. The stratigraphy and micropalaeontology of the Cretaceous Brenton Formation and the PB-A/1 well, near Knysna, Cape Province. Transactions of the Geological Society of South Africa 79 (3): 341-370.

McMillan, I.K. 2003. The Foraminifera of the Late Valanginian to Hauterivian (Early Cretaceous) Sundays River Formation of the Algoa Basin, eastern Cape Province, South Africa. Annals of the South African Museum 106: 1-274.

Mitchum, R.M.; Uliana, M.A.1985. Seismic stratigraphy of carbonate depositional sequences, Upper JurassicLower Cretaceous, Neuquén Basin, Argentina. In Seismic stratigraphy II: an integrated approach to hydrocarbon exploration (Berg, O.R.; Woolverton, D.G.; editores). American Association Petroleum Geologists, Memoir 39: 55-274.

Moore, R.; Pitrat, C. 1961 (Editores). Treatise on Invertebrate Paleontology. Part Q Arthropoda 3. Geological Society of America and University of Kansas Press: 442 p. Lawrence.

Müller, G.W. 1894. Die Ostracoden des Golfes von Neapel und der angrenzenden Meeres-abschnitte. Fauna und Flora Neapel, Monographie (21): 1- 404. Berlin.

Murray, J. W. 1991. Ecology and Palaeoecology of Benthic Foraminifera. Longman Scientific and Technical: 397 p. Nueva York.

Musacchio, E.A. 1979. Datos paleobiogeográficos de algunas asociaciones de foraminíferos, ostrácodos y carófitas del Jurásico medio y el Cretácico inferior de Argentina. Ameghiniana 16 (3-4): 247-271.

Nöel, D. 1958. Étude de coccolithes du Jurassique et du Crétacé inférieur. Publications du Service de la Carte Géologique de l' Algerie (Nouvelle Serie) 2 (20): p. 155-196.

Nöel, D. 1965. Sur les coccolithes du Jurrasique Europeen et d'Afrique du Nord. Essai de classification des coccolithes fossils. Centre Nationale de Recherches Scientifique: 209 p.

Noth, R. 1951. Foraminiferen aus Unter-und Oberkreide des oesterreichischen Anteils an Flysch, Helvetikaum end Vorlandvorkommen. Jahrbuch der Geologischen Bundesanstalt 3: 1-91.

Perch-Nielsen, K. 1968. Der Feinbau und die Klassifikation 
aus dem Maastriktien von Dänemark. Detkonkelige Danske Videnskabernes Selskab Biologiske Skrifter, 16 (1): 1-96.

Perch-Nielsen, K. 1984. Validation of new combinations. INA Newslatter 6: 42-46.

Rahman, A.; Roth, P.H. 1991. Upper Jurassic calcareous nannofossils from the DSDP Site 534 in the Blake Bahama Basin, western North Atlantic. Eclogae Geologica Helvetiae 84 (3): 765-789.

Reinhardt, P. 1964. Finge Kalkfeinige KalkflagellatenGattungen (Coccolithophoriden, Coccolithineen) aus dem Mesozoikum Deutschlands. Monatsberichte der Deutschen Akademie der Wissenschaften zu Berlin 6: 749-759.

Reinhardt, P. 1965. Neue Familien für fossile Kalkflagellaten (Coccolithophoriden, Coccolithineen). Monatsberichte der Deutschen Akademie der Wissenschaften zu Berlin 7: 30-40.

Reinhardt, P. 1966. Fossile vertreter coronoider und styloider Coccolithen (Family Coccolithaceae Poche 1913). Monatsberiche der Deustchen Akademie der Wissenschaften zu Berlin 8: 513-524.

Reinhardt, P. 1967. Fossile coccolithen mit rhagoidem Zentralfeld (Fam. Ahmuellerellaceae, Subord. Coccolithineae). Neues Jahrbuch für Geologie und Paläontologie, Monatshefte (3): 163-178.

Riegraf, W. 1989. Benthonische Schelf-Foraminiferen aus dem Valanginium-Hauterivium (Unterkreide) des Indischen Ozeans südwestlich Madagaskar (Deep Sea Drilling Project Leg 25, Site 249). Geologische Rundschau 78 (3): 1047-1061.

Riegraf, W.; Luterbacher, H. 1989. Benthonische Foraminiferen aus der Unterkreide de 'Deep Sea Drilling Project' (Leg 1-79). Geologische Rundschau 78 (3): 1063-1120.

Reuss, A.E. 1845. Die Versteinerungen der böhmischen Kreideformation. Sttutgart, E. SchweizerbartAbteilung 1: $1-58$.

Reuss, A.E. 1860. Die Foraminiferen der Westphälischen Kreideformation. Sitzungsberichte der Kaiserlichen Akademie der Wissenschaften in Wien, MathematischNaturwissenschaftliche Classe 42: 355-370.

Reuss, A.E. 1863. Die Foraminiferen des norddeutschen Hills und Gault. Sitzungsberichte der Kaiserlichen Akademie der Wissenschaften in Wien, MathematischNaturwissenschaftliche Classe 46 (1): 5-100.

Roemer, F. 1838. Die Cephalopoden des Nord-Deutschen tertiären Meersandes. Neues Jahrbuch für Mineralogie, Geognosie, Geologie und Petrefakten-Kunde: 381-394.

Roemer, F. 1842. Neue Kreide-Foraminiferen. Neues Jahrbuch für Mineralogie, Geognosie, Geologie und Petrefakten-Kunde: 272-273.

Rossi, G.C. 2001. Arenisca Avilé. Facies, ambiente sedimentario y estratigrafía de una regresión forzada del Hauteriviano inferior de la cuenca Neuquina. Tesis Doctoral No. 770 (Inédito), Facultad de Ciencias
Naturales y Museo, Universidad Nacional de la Plata: $331 \mathrm{p}$.

Roth, P. 1978. Cretaceous nannoplankton biostratigraphy and oceanography of the northwestern Atlantic Ocean. Initial Reports of the Deep Sea Drilling Project 44: 731-760.

Sagasti, G. 2002. Estudio sedimentológico y de estratigrafía secuencial de las sedimentitas carbonáticas de la Formación Agrio (Cretácico inferior), en el sector surmendocino de la cuenca Neuquina, República Argentina. Tesis Doctoral No. 764 (Inédito), Universidad Nacional de la Plata, Facultad de Ciencias Naturales y Museo: $280 \mathrm{p}$.

Sagasti, G.; Ballent, S. 2002. Caracterización microfaunística de una transgresión marina: Formación Agrio (Cretácico inferior), Cuenca Neuquina, Argentina. Geobios 35 (6): 721-734.

Sars, G.O. 1925(1922-1928). An account on the Crustacea of Norway. Bergen Museum 9 Crustacea, parts XV, $\mathrm{XVI}: 277 \mathrm{p}$.

Schwarz, E. 2003. Análisis paleoambiental y estratigrafía secuencial de la Formación Mulichinco (Valanginiano), en el sector septentrional de la provincia del Neuquén, Cuenca Neuquina, Argentina. Tesis Doctoral No. 790 (Inédito), Universidad Nacional de la Plata, Facultad de Ciencias Naturales y Museo: 304 p.

Simeoni, M. 1985. Foraminíferos del Cretácico inferior en los niveles basales de la Formación Agrio, Perfil El Marucho, Neuquén, Argentina. Ameghiniana 21 (24): $285-293$.

Simeoni, M. 1988. Foraminíferos del Cretácico Inferior de la Formación Agrio en el perfil Villa del Agrio, Cuenca del Neuquén, Argentina. In Congreso Argentino de Paleontología y Bioestratigrafía, No. 4, Actas 3: 145162. Mendoza.

Simeoni, M. 2000. Zonación bioestratigráfica preliminar del Jurásico superior y Cretácico inferior en la Cuenca Neuquina, centro oeste de Argentina, basada en foraminíferos bentónicos. In Congreso Geológico Chileno, No. 9, Actas 1, sección temática 3: 559-562. Puerto Varas.

Simeoni, M. 2001. Cambios paleobiológicos próximos al límite Jurásico/Cretácico basados en microfósiles calcáreos de Patagonia septentrional. Tesis doctoral (Inédito), Universidad Nacional de la Patagonia San Juan Bosco, Facultad de Ciencias Naturales: 223 p. Comodoro Rivadavia.

Simeoni, M.; Musacchio, E. A. 1996. Cretaceous calcareous microfossils from southern South America: palaeobiogeographic relationships. Zentralblatt für Geologie und Paläontologie 1 (11-12): 1403-1420.

Sollas, W.J. 1877. On the genus Webbina, with descriptions of two new species from the Cambridge Greensland. Geological Magazine 14 (3): 102-105.

Spalletti, L.A.; Poiré, D.; Pirrie, D.; Matheos, S.; Doyle, P. 2001. Respuesta sedimentológica a cambios en el nivel de base en una secuencia mixta clástica- 
carbonática del Cretácico de la Cuenca Neuquina, Argentina. Revista Sociedad Geológica de España 14 (1-2): 57-74.

Stipanicic, P.N.; Rodrigo, F.; Bauliés, O.; Martínez, C. 1968. Las formaciones presenonianas en el denominado Macizo Nordpatagónico y regiones adyacentes. Revista de la Asociación Geológica Argentina 23 (2): 67-98.

Stover, L. 1966. Cretaceous coccoliths and associated nannofossils from France and the Netherlands. Micropaleontology 12 (2): 133-167.

Stradner, H. 1963. New contributions to Mesozoic stratigraphy by means of nannofossils. Proceedings of the World Petroleum Congress, No. 6, Section I, Paper 4: 167-183.

Sturrock, S.; Murray, J.W. 1981. Comparison of low energy and high energy marine middle shelf foraminiferal faunas, Celtic Sea and western English Channel. In Micropalaeontology of Shelf Seas (Neale, J. W.; Brasier, M.; editores). Ellis Horwood: 250-260. Chichester.

Sylvester-Bradley, P.C. 1948. Bathonian Ostracoda from the Boueti Bed of Kangton Harring, Dorset. Geological Magazine 85 (4): 185-204.

Thierstein, H. 1971. Tentative Lower Cretaceous calcareous nanoplankton zonation. Eclogae Geologica Helvetiae 64 (2): 459-488.

Thierstein, H. 1973. Lower Cretaceous Calcareous nannoplankton biostratigraphy. Abhandlungen der Geologischen Bundesanstalt 29: 52 p.

Tucker, M.E. 1991. Sedimentary Petrology. An introduction to the origin of sedimentary rocks. Blackwell Science, Second edition: $260 \mathrm{p}$.

Manuscrito recibido: Septiembre 2, 2005; aceptado: Noviembre 8, 2005.
Tucker, M.E.; Calvet, F.; Hunt, D. 1993. Sequence stratigraphy of carbonate ramps: systems tracts, models and applications to the Muschelkalk carbonate platforms of eastern Spain. International Association of Sedimentologists, Special Publication 18: 397415.

Valicenti, V.H.; Stephens, J.M. 1984. Ostracods from the Upper Valanginian and Upper Hauterivian of the Sundays River Formation, Algoa Basin, South Africa. Revista Española de Micropaleontología 16 (1-3): 171-239.

Whatley, R. 1983. The application of Ostracoda to palaeoenvironmental analysis. In Applications of Ostracoda (Maddocks, R.; editor). University of Houston Geosciences: 51-77. Houston.

Wind, H.; Cepek, P. 1979. Lower Cretaceous calcareous nannoplankton from DSDP Hole 397 A (Northwest African margin). Initial Reports of the Deep Sea Drilling Project 47 (Part I): 221-255.

Wise, S.W.; Wind, F.H. 1977. Mesozoic and Cenozoic calcareous nannofossils recovered by DSDP Leg 36 drilling on the Falkland Plateau, Southwest Atlantic sector of the Southern Ocean. In Reports of the Deep Sea Drilling Project (Barker, P. F.; Daziel, I.W.D. et al.; editors). U.S. Government Printing Office 36: $p$. 269-491.

Worsley, T.R. 1971. Calcareous nannofossil zonation of Upper Jurassic and Lower Cretaceous sediments from the Western Atlantic. In Proceedings of the Second Planktonic Conference, Roma, 1970 (Farinacci, A.; editor). Edizioni Tecnoscienza 2: 13011321. 
LÁMINAS 1-4 


\section{LAMINA 1}

\footnotetext{
(Microfotografías de nanofósiles calcáreos hallados en la sección Cuesta del Chihuido, Cuenca Neuquina, Argentina, tomadas con nícoles cruzados y 1562,5 aumentos; la barra representa $2 \mu \mathrm{m}$ )
}

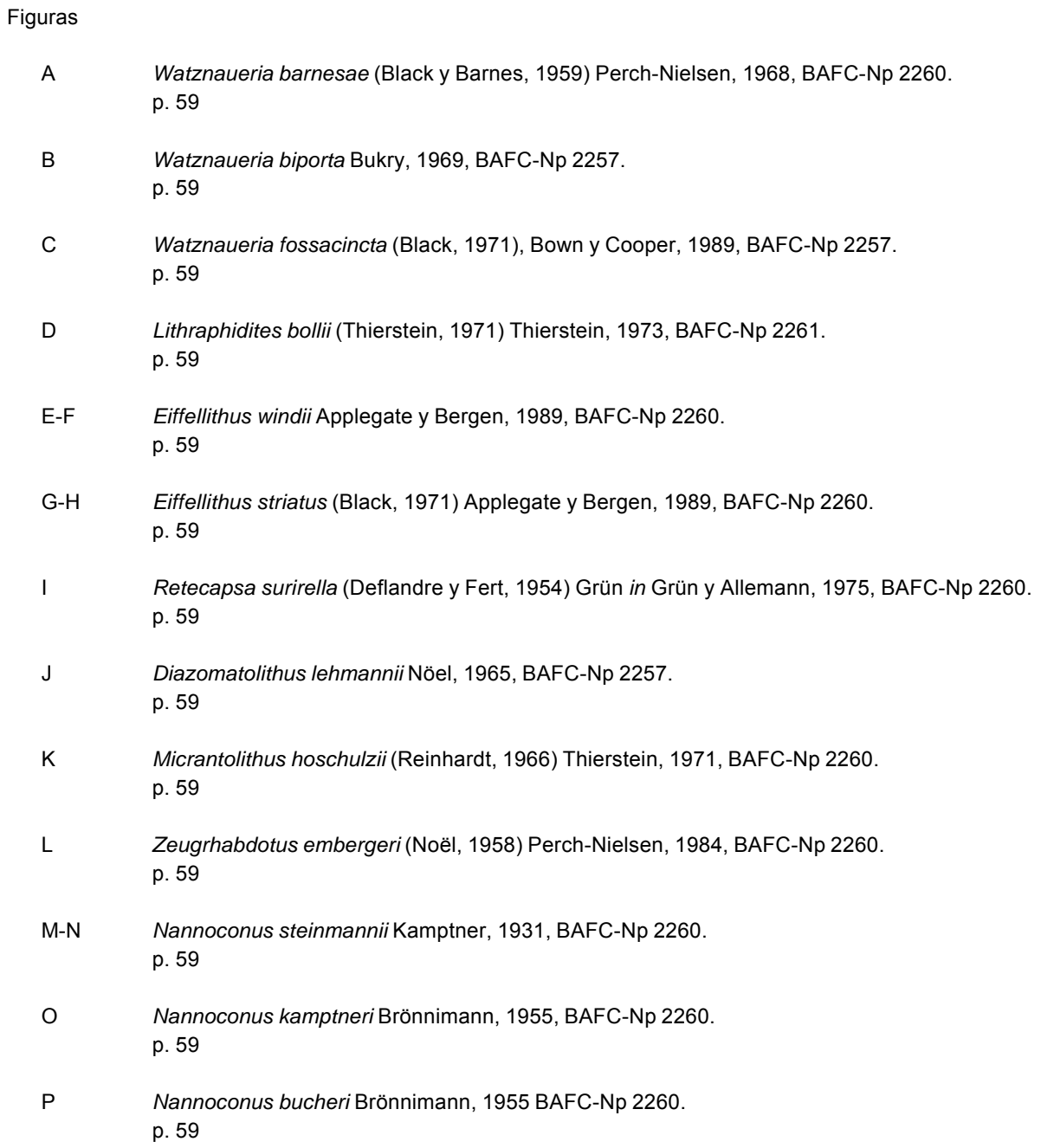



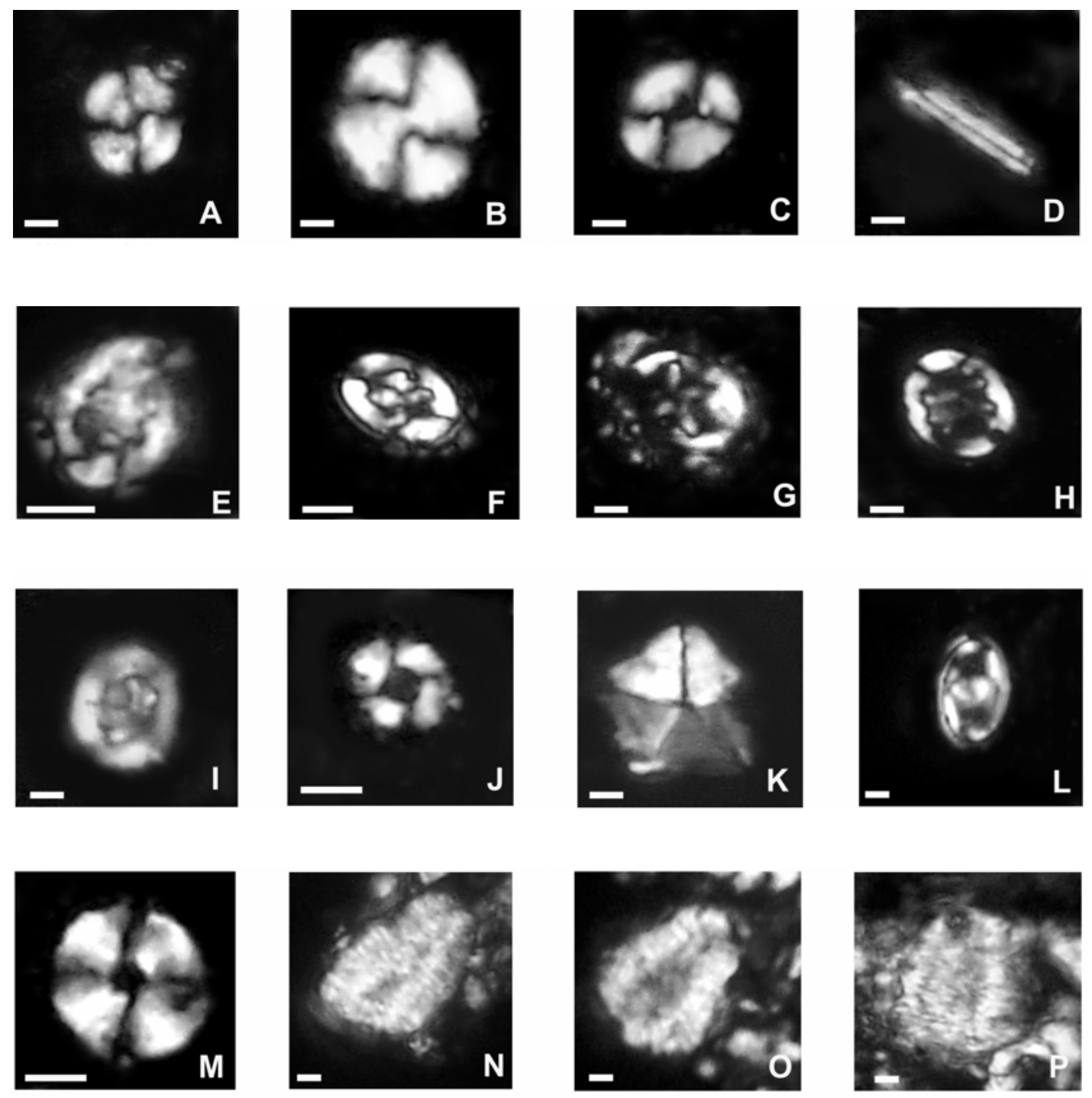
LÁMINA 2

(Foraminiferos de la sección Cuesta del Chihuido, Cuenca Neuquina, Argentina; escala: A-D, G= 0, 500 mm; F, H, J-W= 0,100 mm; I, aprox. 18x.)

Figuras

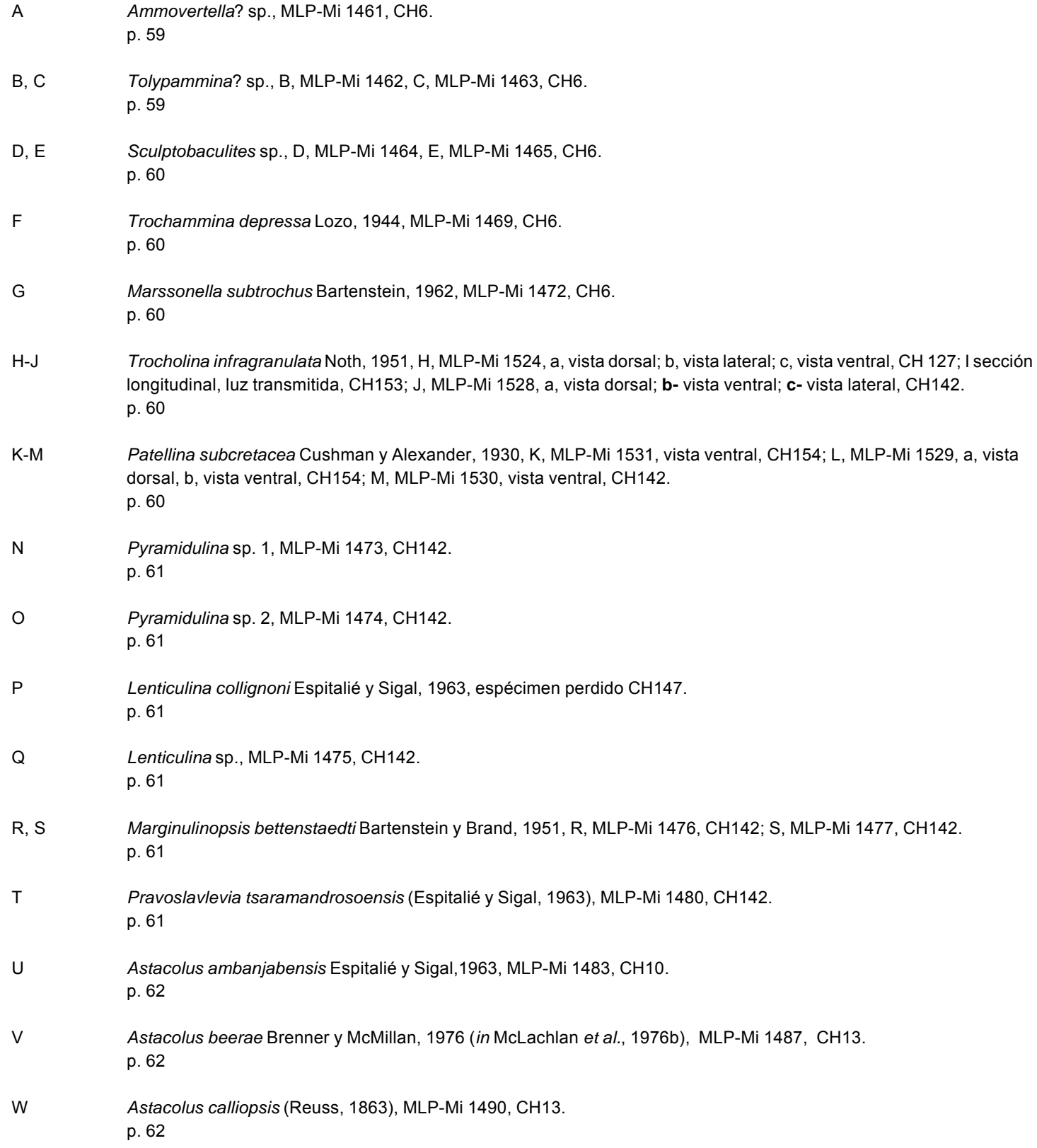


LÁMINA 2

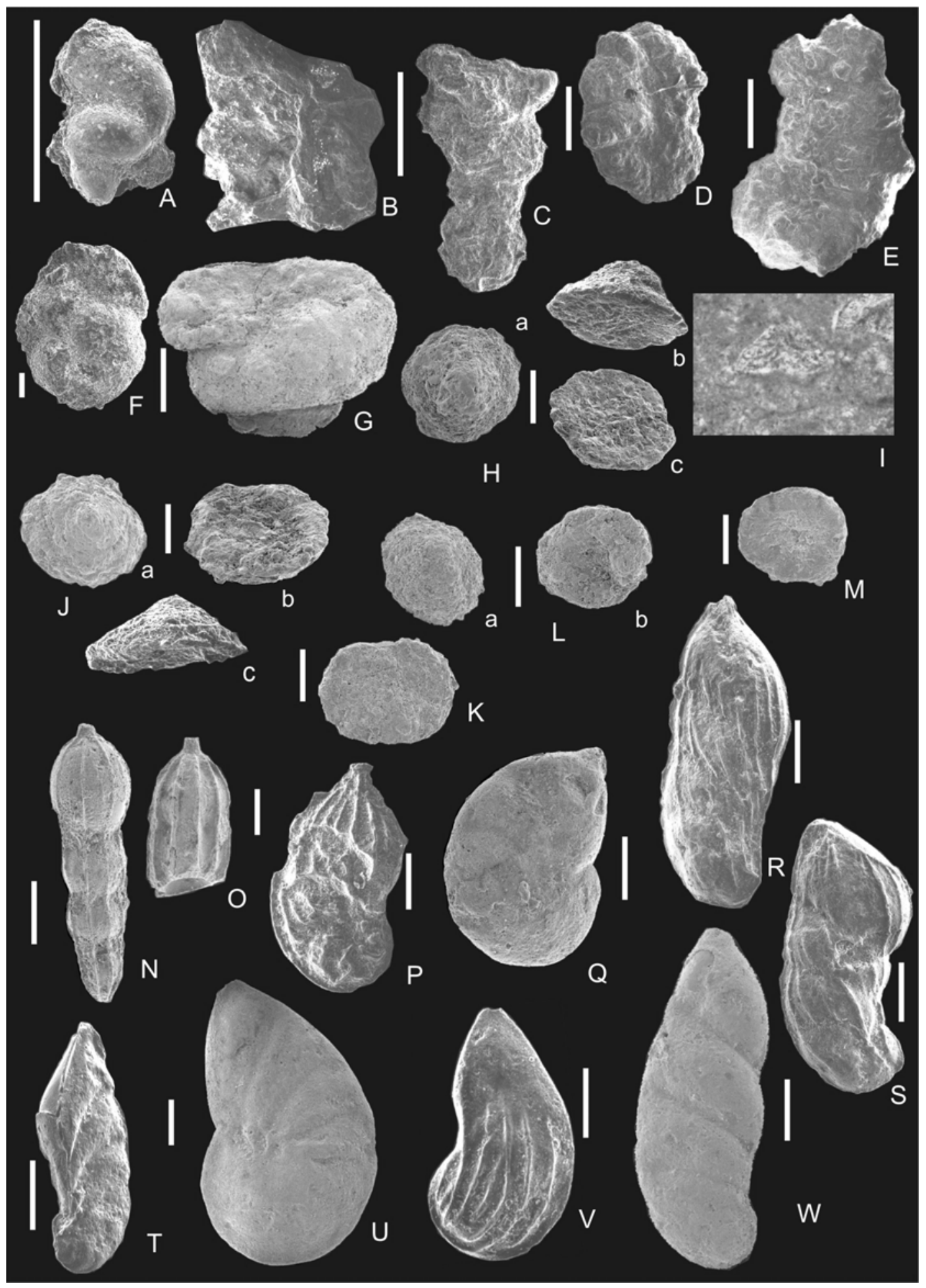




\section{LÁMINA 3}

(Foraminiferos de la sección Cuesta del Chihuido, Cuenca Neuquina, Argentina; Escala= 0, 100 mm, Q, aprox. 8x.)

Figuras

A Marginulina schloenbachi (Reuss, 1863), MLP-Mi 1491, CH142.

p. 62

B Marginulina? sp., MLP-Mi 1487, CH13.

p. 62

C-D Vaginulinopsis matutina (d'Orbigny, 1849), C, MLP-Mi 1494, CH142; D, MLP-Mi 1493, CH142. p. 62

E Vaginulinopsis sp., MLP-Mi 1495, CH142

p. 62

F $\quad$ Citharina sparsicostata (Reuss, 1951) sensu Bartenstein y Brand, MLP-Mi 1498, CH13. p. 63

G Citharina cristellarioides (Reuss, 1845) sensu Bartenstein y Brand, 1951, MLP-Mi 1500, CH13. p. 63

H, I Planularia complanata (Reuss, 1845), H, MLP-Mi 1502, CH142, I, MLP-Mi 1501, CH142. p. 63

J Citharina intumescens var. culter (Fursenko y Polenova, 1950) sensu Espitalié y Sigal,1963, MLP-Mi 1496, CH13. p. 62

K, L Planularia crepidularis Roemer, 1842, K, MLP-Mi 1504, CH13; L, MLP-Mi 1503, CH13. p. 63

M Reussoolina? sp., MLP-Mi 1509, $\mathrm{CH} 142$.

p. 63

$\mathrm{N}$

Globulina lacrima (Reuss, 1845), MLP-Mi 1510, CH142.

p. 64

O-Q Guttulina sp.; O, MLP-Mi 1512, CH142; P, MLP-Mi 1513, CH142; Q sección transversal, CH153. p. 64

R Globulina prisca (Reuss, 1863), MLP-Mi 1511, CH142.

p. 64

S Pyrulina cylindroides (Roemer, 1838), MLP-Mi 1517, CH142.

p. 64 
LÁMINA 3

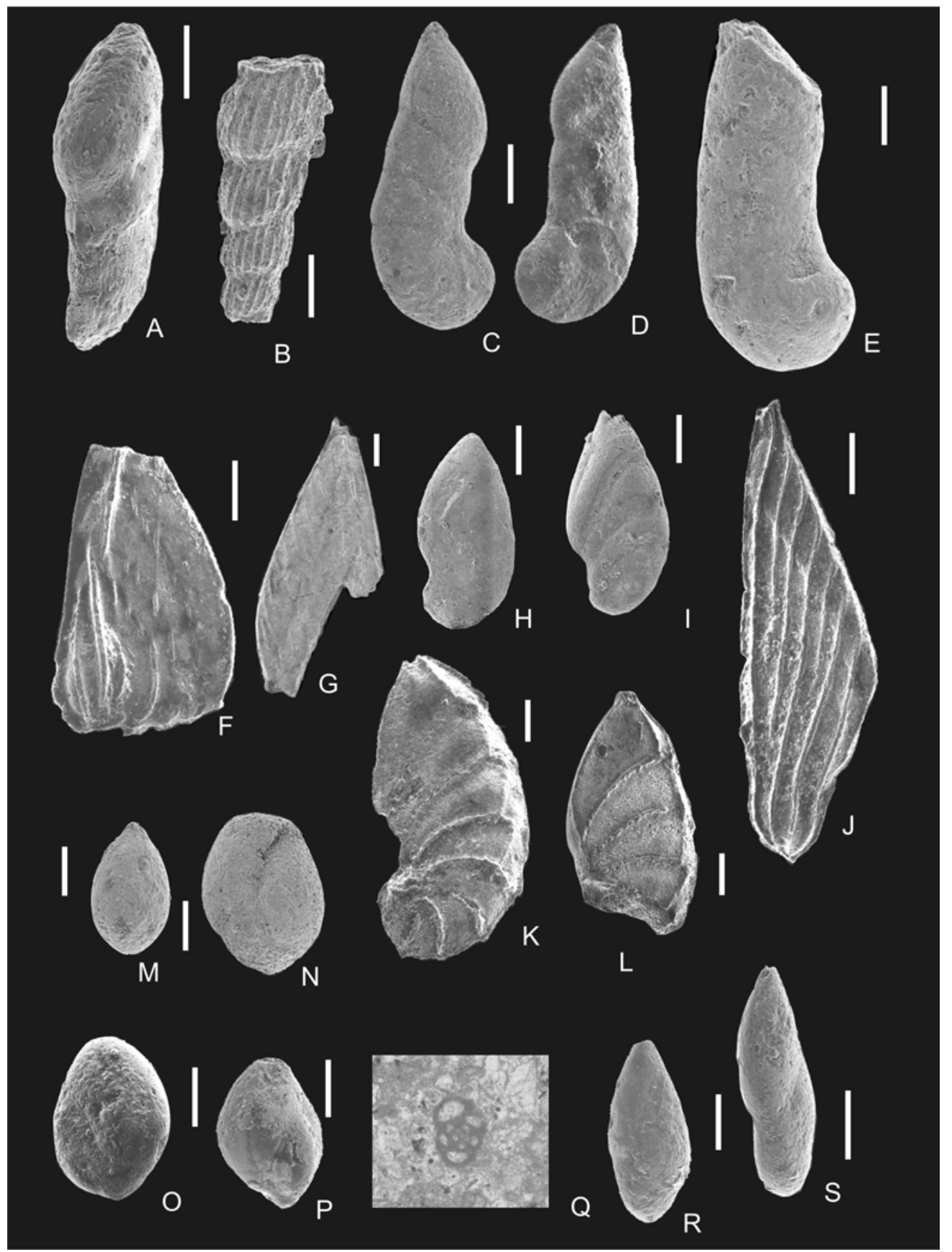




\section{LÁMINA 4}

(Foraminíferos y ostrácodos de la sección cuesta del Chihuido, Cuenca Nequina, Argentina; escala= 0, $100 \mathrm{~mm}$ ).

Figuras

A Psilocitharella arguta (Reuss, 1860), MLP-Mi 1508, CH142.

p. 63

B, C Bullopora laevis (Sollas, 1877), B, espécimen perdido, CH 6; C, MLP-Mi 1518, CH 6.

p. 64

D Webbinella? sp., MLP-Mi 1521, CH 147

p. 64

E Epistomina loncochensis Ballent, 2002 (in Sagasti y Ballent, 2002), MLP-Mi 1522, CH 3.

p. 65

F Rostrocytheridea sp. nov.?, MLP-Mi 1424, CH147, caparazón femenino, vista lateral derecha. p. 65

G Mandelstamia? sp., MLP-Mi 1433, CH147, caparazón, vista lateral izquierda. p. 65

$\mathrm{H}$ Procytherura sp. nov.?, MLP-Mi 1435, CH6, caparazón, vista lateral izquierda. p. 65

I Majungaella pavta Ballent, Ronchi y Whatley, 1998, MLP-Mi 1443, caparazón, vista lateral derecha. p. 66

J Sondagella colchesterensis Valicenti y Stephens, 1984, MLP-Mi 1446, caparazón, vista lateral izquierda. p. 66

K Sondagella theloides Dingle, 1969, MLP-Mi 1445, CH10, caparazón, vista lateral derecha p. 66 


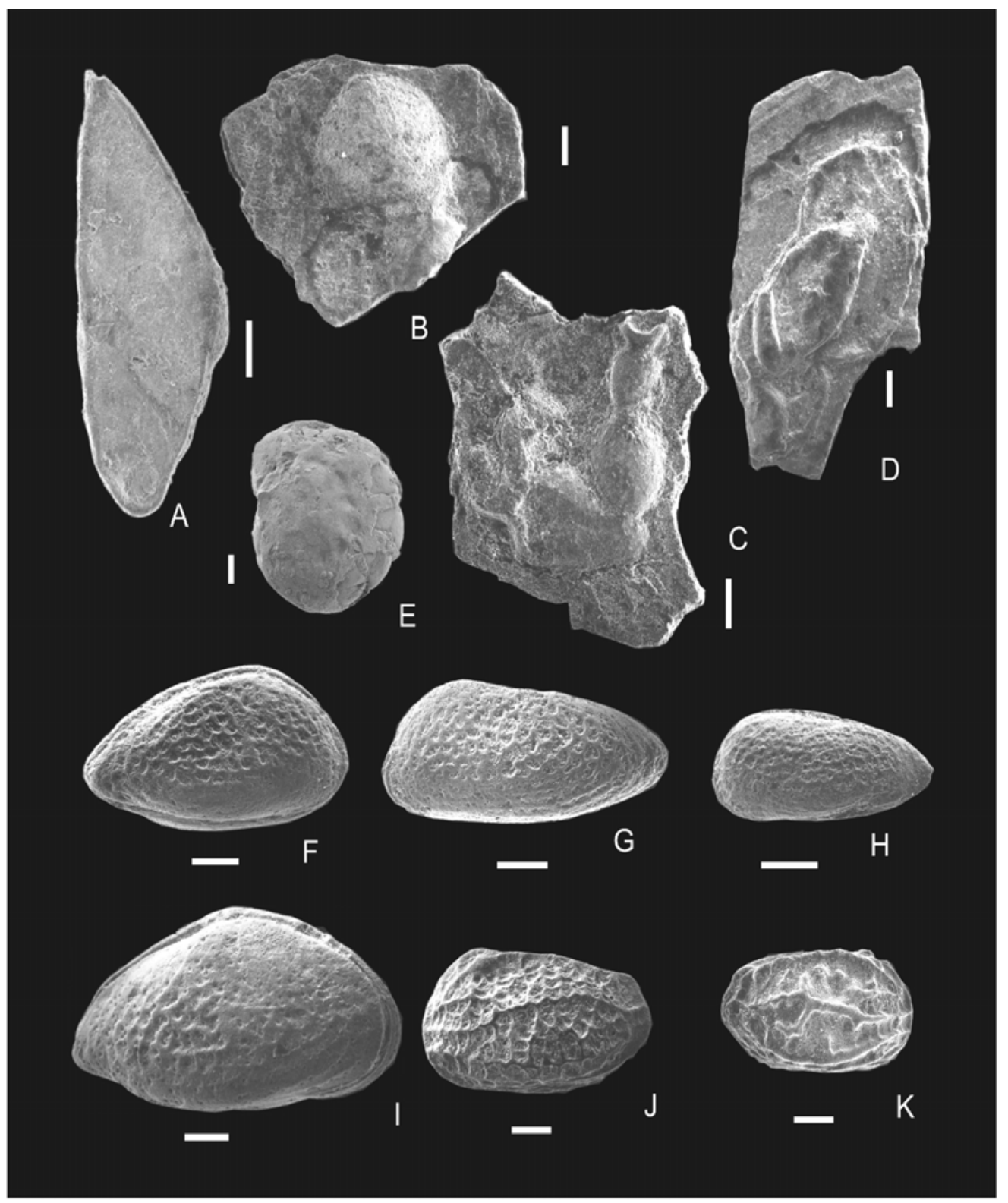

